TRANSACTIONS OF THE

AMERICAN MATHEMATICAL SOCIETY

Volume 302, Number 2, August 1987

\title{
DEGREES OF SPLITTINGS AND BASES OF RECURSIVELY ENUMERABLE SUBSPACES
}

\author{
R. G. DOWNEY, J. B. REMMEL AND L. V. WELCH
}

\begin{abstract}
This paper analyzes the interrelationships between the (Turing) of r.e. bases and of r.e. splittings of r.e. vector spaces together with the relationship of the degrees of bases and the degrees of the vector spaces they generate. For an r.e. subspace $V$ of $V_{\infty}$, we show that $\alpha$ is the degree of an r.e. basis of $V$ iff $\alpha$ is the degree of an r.e. summand of $V$ iff $\alpha$ is the degree and dependence degree of an r.e. summand of $V$. This result naturally leads to explore several questions regarding the degree theoretic properties of pairs of summands and the ways in which bases may arise.
\end{abstract}

1. Introduction. One of the most fundamental and pervasive questions arising from recursive model theory is that of the relationship between the degree of an r.e. structure and the degrees of its r.e. sets of generators. For r.e. sets this question is, of course, quite trivial. However, for the structures we shall consider (namely r.e. subspaces), this question turns out to be quite complex. The goal of this paper is to analyze the following question:

(1.1) For $V \in L\left(V_{\infty}\right)$, what can be said about the relationships between $B(V)$, the collection of degrees of r.e. bases of $V, S(V)$, the degrees of halves of splittings of $V$ by direct sum, and $d(V)$, the Turing degree of $V$ as a set?

(Henceforth, we assume the reader to be familiar with $L\left(V_{\infty}\right)$ and only give a brief review of notations and terminology in §2.)

Now already some partial results concerning (1.1) are known. We shall review and extend some of these in $\S 3$. These tend to fall into three categories: the first category consists of those splitting results which show that analogues of results from r.e. sets hold in $L\left(V_{\infty}\right)$. For example, Retzlaff [Rt] shows the analogue of Friedberg's splitting theorem holds in $L\left(V_{\infty}\right)$. The second category consists of those results which show that $L\left(V_{\infty}\right)$ has certain splitting features not to be found in $L(\omega)$. For example, Ash and Downey [AD] show that given any $V \in L\left(V_{\infty}\right)$ we may find decidable subspaces $V_{1}, V_{2} \in L\left(V_{\infty}\right)$ with $V_{1} \oplus V_{2}=V$ and hence, in particular, we can have $d\left(V_{1}\right) \vee d\left(V_{2}\right) \neq d(V)$, although the $V_{i}$ split $V$.

The final category of results consists of some observations due to Remmel, connecting $B(V)$ and $S(V)$. The archetype of such known results is showing that $S(V) \subseteq B(V)$. It is shown in Remmel [Re1] that a very easy way to manufacture bases is as follows: Let $V \in L\left(V_{\infty}\right)$ and $V_{1} \oplus V_{2}=V$ be an r.e. splitting of $V$. Then

Received by the editors September 23, 1985 and, in revised form, July 15, 1986.

1980 Mathematics Subject Classification (1985 Revision). Primary 03D45, 03D25, 03D30.

Some of these results we obtained whilst the first author was at the National University of Singapore and partially supported by Singapore Grant RP-85/83. The second author was supported by NSF Grant MCS-8202333. 
Remmel [Re1] showed that $V_{1}$ has an r.e. basis $R \equiv_{T} V_{1}$ and by Dekker [De], $V_{2}$ has a recursive basis $R_{2}$. Consequently $R_{1} \cup R_{2}$ is an r.e. basis of $V$ of degree $d\left(V_{1}\right)$.

Our main result of $\S 4$ is to show that the reverse inclusion also holds. In fact, we show that $\alpha$ is the degree of an r.e. basis of $V$ iff $\alpha$ is the degree of an r.e. summand of $V$ iff $\alpha$ is the dependence degree and degree of an r.e. summand of $V$. This result has several interesting consequences, one of which is a complete analogue of Sacks' splitting theorem simultaneously for degrees and dependence degrees (extending Shore $[\mathbf{S h}])$.

Because of these results, we may treat $B(V)$ and $S(V)$ as the same, and henceforth we shall concentrate mainly on splittings rather than bases. It follows therefore, that bases come essentially from the Remmel process. This leads to a number of questions concerning what types of splittings bases come from.

One reasonable conjecture (noted by several authors) supported by $\S 4$, is that for fully extendible subspaces $V$, we can reduce questions about $B(V)$ to questions about splittings of a fixed r.e. basis of $V$. Thus let $V=(R)^{*}$ where $R$ is an r.e. subset of a recursive basis of $V_{\infty}$. Formally, we shall say that $V$ has (the) basis reflection property (BRP) if, given any r.e. basis $B$ of $V$ there is an r.e. splitting $R_{1} \sqcup R_{2}$ of $R$, such that $R_{1} \equiv_{T} B$.

For fully extendible r.e. subspaces, life would be very easy, if every such subspace had BRP; for then questions about $B(V)$ and $S(V)$ would reduce to ones about the lattice of r.e. sets.

However, in $\S 5$ our main results are that if $\delta$ is any nonzero r.e. degree, then $\delta$ contains r.e. subspaces both with and without BRP.

Our remaining hope is that every r.e. basis comes from a splitting of some basis of the same degree as $V$. This is one of the basic properties of r.e. sets, namely that if $A_{1} \sqcup A_{2}=A$ is an r.e. splitting of $A$, then $d\left(A_{1}\right) \vee d\left(A_{2}\right)=d(A)$. Already we have seen that the direct analogue fails for $L\left(V_{\infty}\right)$. In $\S 7$ we show that any reasonable extension will fail by constructing an r.e. basis $B$ of an r.e. subspace $V$ such that whenever $Q \oplus R=V$ is an r.e. splitting of $V$ with $Q \equiv_{T} B$, it is always the case that $d(Q) \vee d(R) \neq d(V)$. This means, in particular, we cannot reduce questions about splittings of $V \in L\left(V_{\infty}\right)$ to even degree theoretic ones about the structure of the r.e. degrees.

The proof of this result uses a class of r.e. subspaces, the strongly atomic ones, whose r.e. set analogues have proved very useful in studying splitting properties of r.e. sets and degree embeddings (cf. [DW, AS2]). $V \in L\left(V_{\infty}\right)$ is called strongly atomic if, whenever $Q \oplus W=V$ is an r.e. splitting of $V$, then $\inf \{d(Q), d(W)\}=\mathbf{0}$.

In $\S 6$ we give a construction of a strongly atomic r.e. subspace. Indeed, we construct a high r.e. subset $R$ of recursive basis $B$ of $V_{\infty}$ such that if $W \in L\left(V_{\infty}\right)$ and $W \oplus(B-R)^{*}=V_{\infty}$, then $W$ is strongly atomic. Combining this result with several from the literature, will yield many lattice theoretic existence theorems for strongly atomic r.e. subspaces, and some "antisplitting" results.

To get the result of $\S 7$, we then modify the $\S 6$ construction with some properties of weak truth table degrees ( $W$-degrees). Specifically, we construct an r.e. subset $R$ of a recursive basis $B$ of $V_{\infty}$ and an r.e. nonrecursive basis $Q$ of $(R)^{*}$, such that

(i) $(R)^{*}$ has contiguous degree (that is, contains only one r.e. $W$-degree),

(ii) $(R)^{*}$ is strongly atomic, 
(iii) $Q$ is a $W$-anticupping witness for $R$.

As we show in $\S 7$, these properties will suffice.

Finally, in $\S 8$ we study the $m$-degrees of r.e. bases. Apart from their intrinsic interest, we feel that these are important because of Guichard's classification [Gu1] of the automorphisms of $L\left(V_{\infty}\right)$ as those induced by recursive invertible semilinear transformations of $V_{\infty}$. One interesting problem for $L\left(V_{\infty}\right)$ is to find any reasonable orbits. Because of Guichard's work, any such orbit must preserve the $m$-degrees of bases. Hence in the " $L\left(V_{\infty}\right)$ " setting, reducibilities such as 1 - or $m$-reducibilities, will be much more important when studying automorphisms, than they are in the lattice of r.e. sets.

The authors wish to thank Michael Stob and K. Ambos-Spies for many useful conversations concerning the subject of this paper.

2. Notation and terminology. Let $\left\{\phi_{i}: i \in \omega\right\}$ be an effective list of all the partial recursive functions, and $\left\{\Phi_{e}: e \in \omega\right\}$ an effective list of all oracle machines. We shall write $\Phi_{e, s}(A ; z)$ for the result, if any, of performing $s$ steps in the computation of the oracle machine $\Phi_{e}$ with oracle $A$ and input $z$. If this halts in $s$ or fewer steps we write $\Phi_{e, s}(A ; z) \downarrow$ and $\Phi_{e, s}(A ; z) \uparrow$ otherwise. Thus $\Phi_{e}(A)=B$ means for all $z, \Phi_{e}(A ; z) \downarrow$ and equals $B(z)$. Here we are confusing sets with their characteristic functions. We shall use the standard use function defined as

$$
u\left(\Phi_{k}(A ; x)\right)=\left\{\begin{array}{c}
\mu y \&\left(y \geq x \text { and the computations of } \Phi_{k}(A ; x) \downarrow\right. \\
\text { and } \left.\Phi_{k}(A[y] ; x) \text { are identical }\right) \text { if } y \text { exists } \\
\text { undefined otherwise, }
\end{array}\right.
$$

where $A[y]=\{x \in A \mid x \leq y\}$. By convention, we presuppose that all computations involving r.e. sets are bounded by $s$ at stage $s$.

In this paper, we shall use three reducibilities: $\leq_{T}$ (Turing), $\leq_{m}$ (many-one), and $\leq_{W}$ (weak truth table). The first two are well known and we remind the reader that $A \leq_{W} B$ means that there is an $i$ and a $j$ with $\phi_{j}$ total such that

$$
\text { for all } x, \quad \Phi_{i}(B)=A \text { and } u\left(\Phi_{i}(A ; x)\right) \leq \phi_{j}(x) .
$$

Intuitively, this means that there is a recursive bound on the information used in the $\Phi_{i}$-computation from $B$ to $A$. An excellent reference for $W$-degrees is Stob [St]. We shall draw from Stob [St] and Ladner and Sasso [LS]. In the obvious way, we shall specify $\Delta-\operatorname{deg}(A)$ for the $\Delta$-degree of $A$ where $\Delta$ is $T$-, $m$ - or $W$-. A couple of degree theoretic concepts we shall need are as follows. An r.e. $T$-degree $\delta$ is called contiguous if whenever $A$ and $B$ are r.e. of degree $\delta$ then $B \equiv_{T} A$ implies $B \equiv_{W} A$. In particular if $A$ is contiguous (i.e. $A$ is an r.e. set of contiguous degree) then for all $B \leq_{T} A, B \leq_{W} A$. If $A$ is an r.e. nonrecursive set then there exists an r.e. set $B$ with $\varnothing<_{T} B<_{W} A$ such that $B$ has contiguous degree. This result is due to Ladner and Sasso [LS]. Certainly not every r.e. set has contiguous degree. If $\delta$ is an r.e. contiguous degree, then $\delta$ is low ln $_{2}$ in high/low hierarchy, moreover every nonzero r.e. degree has a noncontiguous r.e. predecessor (cf. [LS]).

An r.e. $\Delta$-degree $\delta$ is said to $\Delta$-cup to an r.e. $\Delta$-degree $\alpha$ if $\delta<_{\Delta} \alpha$ and there exists an r.e. $\Delta$-degree $\beta$ such that $\beta \equiv_{\Delta} \alpha$ and $\Delta$-sup $(\delta, \beta)=\alpha$. An r.e. $\Delta$-degree $\delta$ is said to have the $\Delta$-anticupping property if it has a $\Delta$-predecessor which does not $\Delta$-cup to $\delta$. Ladner and Sasso have shown every r.e. $W$-degree has the $W$ anticupping property and so each contiguous r.e. $T$-degree has the $T$-anticupping 
property (cf. [LS]). Harrington [Ha] has shown that each high r.e. T-degree has the anticupping property via a high anticupping witness (with the appropriate meaning). Finally an r.e. degree $\alpha$ is called branching if there exist r.e. degrees $\beta, \gamma$ such that $\left.\beta\right|_{\Delta \gamma} \gamma$ and $\Delta-\inf \{\beta, \gamma\}=\alpha$. The existence of minimal pairs shows that $\mathbf{0}$ is a branching degree.

For any unexplained notation and terminology concerning r.e. degrees we refer the reader to Odifreddi $[\mathbf{O d}]$, Soare $[\mathbf{S o 1}, \mathbf{2}]$ and Stob $[\mathbf{S t}]$.

We now give a brief review of some effective linear algebra. The universal object $V_{\infty}$ may be considered as the formal space generated by $e_{i}=\langle 0, \ldots, 0,1,0, \ldots\rangle$ over a recursive field. Its distinguishing characteristics are that,$+ \cdot$, , and $=$ are all recursive and we can decide in a finite number of steps whether or not $x \in$ $\left\{a_{1}, \ldots, a_{n}\right\}^{*}$, where for $A \subset V_{\infty},(A)^{*}$ denotes the subspace generated by $A$. If $V \in L\left(V_{\infty}\right)$, then $D(V)=\{\mathbf{x} \mid \mathbf{x}$ is a $k$-tuple (some $k \in \omega)$ and $\mathbf{x}$ is dependent over $V\}$ is called the dependence set of $V$. We refer to the Turing degree of $D(V)$ as the dependence degree of $V$. If $D(V) \equiv_{T} \mathbf{0}$ we say $V$ is decidable. From Metakides and Nerode [MN2], $V$ is decidable iff there exists $V^{\prime} \in L\left(V_{\infty}\right)$ such that $\left(V \cup V^{\prime}\right)^{*}=V_{\infty}$ and $V \cap V^{\prime}=\{\overrightarrow{0}\}$. In such a case we write $V \oplus V^{\prime}=V_{\infty}$. A subspace $V$ of $V_{\infty}$ is called fully co-r.e. if there exists a recursive basis $B$ of $V_{\infty}$ and a co-r.e. subset $C$ of $B$ with $(C)^{*}=V$. Alternatively "fully co-r.e." means that it is co-r.e. and has a fully extendible basis, i.e. a basis contained in an r.e. basis of $V_{\infty}$. Fully cor.e. subspaces are "natural" complements of r.e. subspaces, and each r.e. subspace has one [Do1, 2]; however, fully co-r.e. subspaces may have many different r.e. complements (see Downey [Do2] and Downey-Remmel [DR1]).

We let $\left\{W_{e}: e \in \omega\right\},\left\{\omega_{e}: e \in \omega\right\}$ and $\left\{I_{e}: e \in \omega\right\}$ be effective listings of r.e. subspaces, r.e. sets and r.e. independent sets respectively, where $W_{e}=\left(I_{e}\right)^{*}$. Define $W_{e, s}=\left\{x \in\left(I_{e, s}\right)^{*} \mid x \leq s\right\}$. By convention $x \in I_{e, s}$ implies $x \leq s$. We denote the dimension of $V$ by $\operatorname{dim}(V)$ and $\operatorname{dim}(V / W)$ denotes the dimension of $(V \cup W)^{*}$ modulo $W$. If $I$ is an independent set, and $x \in(I)^{*}$ then $\operatorname{supp}_{I}(x)$ denotes the support of $x$ relative to $I$, namely the unique smallest finite subset $F$ of $I$ with $x \in(F)^{*}$. We similarly define the support of $x$ relative to $I$ over $V$, if $I$ is independent over $V$. If $V$ is a subspace of $V_{\infty}$ we say $V$ is immune if $\operatorname{dim}(V)=\infty$ and $V$ has no infinite dimensional r.e. subspaces.

Let $V \in L\left(V_{\infty}\right)$ and suppose $\operatorname{dim}\left(V_{\infty} / V\right)=\infty$. We say $V$ is (i) simple if $W \in L\left(V_{\infty}\right)$ and $W \cap V=\{\overrightarrow{0}\}$ implies $\operatorname{dim}(W)<\infty$, (ii) maximal if, for all $W \in L\left(V_{\infty}\right)$ if $W \supset V$ then either $\operatorname{dim}(W / V)<\infty$ or $\operatorname{dim}\left(V_{\infty} / W\right)<\infty$, (iii) $k$-thin if there exists $Q \in L\left(V_{\infty}\right)$ with $\operatorname{dim}\left(V_{\infty} / Q\right)=k$ and $Q \supset V$ and for all $W \in L\left(V_{\infty}\right)$ if $W \supset V$ then either $\operatorname{dim}(W / V)<\infty$ or $W \supset Q$, (iv) supermaximal if $V$ is 0 -thin, that is if $W \supset V$ and $\operatorname{dim}(W / V)=\infty$ then $W=V_{\infty}$, (v) super$r$-maximal if, for $Q, R \in L\left(V_{\infty}\right), Q+R=V_{\infty}$ implies either $Q+V=V_{\infty}$ or $R+V=V_{\infty}$, (vi) nowhere simple if for all $Q \in L\left(V_{\infty}\right)$ if $\operatorname{dim}(Q / V)=\infty$ then there exists $Q^{\prime} \in L\left(V_{\infty}\right)$, with $Q^{\prime} \subset Q, Q^{\prime} \cap V=\{\overrightarrow{0}\}$ and $\operatorname{dim}\left(Q^{\prime}\right)=\infty$, and finally (vii) effectively nowhere simple if in (vi) we can compute an index for $Q^{\prime}$ from one for $Q$. (References for the above: for (i) and (ii) see [MN2], (iii) and (iv) see [KR], (v) see [Gu1] and (vi) and (vii) see [NR1].)

An independent set $I$ is called nonextendible if $\operatorname{dim}\left(V_{\infty} / I^{*}\right)=\infty$ and for all r.e. independent sets $J \supset I$, card $(J-I)<\infty$. For example, any r.e. basis of a supermaximal subspace is nonextendible. If $I$ is not r.e. but is extendible, we say $I$ 
is an $\alpha$-repere (cf. [De]) or sound (cf. [Do2]). A pretty observation from [LR1,2] is that if $B$ is an r.e. basis of $V$ then $B \leq_{W} V$. Any further effective algebra may be found in a survey paper of Nerode and Remmel [NR3], which is a good reference for all of this material.

We have attempted to keep notation and terminology more-or-less standard. We suggest the reader unfamiliar with $V_{\infty}$ identify the underlying field with the rationals (if infinite) or $G F(2)$, the Galois two element field (if finite).

3. Review and extensions. The purpose of this section is to give a brief review (and some extensions of) some results scattered throughout the literature.

Much of the original impetus for studying $L\left(V_{\infty}\right)$ was to see if analogues of results from r.e. sets held in $L\left(V_{\infty}\right)$. The first such splitting theorem was due to Retzlaff [Rt] who showed, in particular, that Friedberg's splitting theorem held in $L\left(V_{\infty}\right)$. He showed that

ThEOREM 3.1 (RETZlafF $[\mathbf{R t}]$ ). Let $V \in L\left(V_{\infty}\right)$ be nondecidable. Then there exist nondecidable $V_{1}, V_{2} \in L\left(V_{\infty}\right)$ such that $V_{1} \oplus V_{2}=V$. Moreover, if $V$ is nonrecursive, the $V_{i}$ may also be chosen to be nonrecursive.

The proof technique is by direct analogue of Friedberg's method, along the lines of Metakides and Nerode's analogue of an $e$-state construction to produce a maximal subspace in [MN2]. Already in [MN1], it was realized that if the underlying field is infinite, recursive but not decidable spaces were possible. Indeed, they showed

ThEOREM 3.2 (METAKIDES AND Nerode [MN1,2]). Suppose $F$ is infinite. Let $V \in L\left(V_{\infty}\right)$; then there exists a recursive $V^{\prime} \subset V$ with $\operatorname{dim}\left(V / V^{\prime}\right) \leq 1$.

We remark that since $V$ and $V^{\prime}$ have the same dependence degrees, choosing $V$ to be nondecidable and considering $V_{\infty} \bmod V^{\prime}$ gives an example of a recursively presented vector space (namely $V_{\infty} \bmod V^{\prime}$ ) with no recursive basis. From our point of view, it also follows that if $V$ were nonrecursive, then $V=V^{\prime} \oplus(\{x\})^{*}$ for some $x \in V-V^{\prime}$ and $d\left(V^{\prime}\right) \vee d\left((\{x\})^{*}\right)=\mathbf{0} \neq d(V)$. We extend Theorem 3.2 for nonrecursive subspaces by showing

THEOREM 3.3. Suppose the field of scalars is infinite. Let $V \in L\left(V_{\infty}\right)$ be nonrecursive. Then there exists an infinite collection $\left\{W_{i}: i \in \omega\right\}$ of r.e. subspaces such that for all $i$,

(a) $W_{i} \subset V$,

(b) $\operatorname{dim}\left(V / W_{i}\right)=1$,

(c) for all $i \neq j,\left.W_{i}\right|_{T} W_{j}$.

PROOF. We shall prove a somewhat simpler statement (and leave a dovetail construction to the reader). We show that if $V$ is as above and $\varnothing<_{T} C \leq_{T} V$ is r.e. then there exists $W \in L\left(V_{\infty}\right)$, such that

(i) $W \subset V$ and $\operatorname{dim}(V / W)=1$,

(ii) $W_{T} \ngtr C$,

(iii) $W \neq_{T} \varnothing$.

We build $W=\bigcup_{s} W_{s}$ in stages, so as to satisfy

$$
N_{e}: \Phi_{e}(W) \neq C, \quad P_{e}: \bar{\omega}_{e} \neq W .
$$


(Recall here that $\omega_{e}$ denotes the $e$ th r.e. set.) It is important to define for an r.e. set $A, \hat{A}_{s}=\left\{y \in\left(A_{s}\right)^{*}: y \leq s\right\}$. To meet the $N_{e}$, we measure the length of agreement

$$
l(e, s)=\max \left\{y \mid \forall x<y\left(\Phi_{e}\left(\hat{W}_{s} ; x\right)=C(x)\right)\right\} .
$$

Corresponding to this is a Sacks' restraint on the use function of the computation above through length $l(e, s)$, that is to the first disagreement. Call this $r(e, s)$ and as usual $R(e, s)=\max _{i \leq e}\{r(i, s)\}$. As usual with algebraic degree arguments, our construction is sensitive to stray linear combinations of elements entering $W_{s+1}-W_{s}$. We therefore say $x$ is $e$-good at stage $s+1$ if $\left(W_{s} \cup\{x\}\right)^{*}[R(e, s)]=\left(W_{s}\right)^{*}[R(e, s)]$. By the use principle and exchange, $e$-good elements protect convergent computations, associated with $N_{j}$ for $j \leq e$.

Via Remmel's [Re1] technique, there is an enumeration of $V$ via an r.e. basis $B=\bigcup_{s} B_{s}$ with $B_{s+1}=\left\{z, b_{0}, \ldots, b_{s}\right\}, B \equiv_{T} V$ and $V_{s+1}=\hat{B}_{s+1}$. We build $W=\bigcup_{s} W_{s}$ in stages so that $\{z\} \oplus W=V$, and so $\operatorname{dim}(V / W)=1$. We say $P_{e}$ is satisfied at stage $s+1$ if $\omega_{e, s} \cap W_{s} \neq \varnothing$. We say $P_{e}$ requires attention at stage $s+1$ if there exists a least $x_{e}$ such that $x_{e}$ is $e$-good, $P_{e}$ is not satisfied, and $x_{e} \in \omega_{e, s} \cap\left(\left(V_{s+1}\right)^{*}-\left(V_{s}\right)^{*}\right)$.

Construction.

Stage 0. Set $r(e, 0)=0$ all $e \in \omega$ and $W_{0}=\{\overrightarrow{0}\}$.

Stage $s+1$. We suppose

(i) $B_{s+1}=\left\{z, b_{0}, \ldots, b_{s}\right\}$,

(ii) $z \notin W_{s}^{*}$, and

(iii) $\left(B_{s+1}\right)^{*}=\left(W_{s} \cup\{z\}\right)^{*}$.

Now if, for all $e \leq s, P_{e}$ does not require attention, find $\lambda_{s}$ in $F$ with

$$
\lambda_{s}=\mu \lambda\left(z+\lambda b_{s} \text { is } s \text {-good at stage } s+1\right) .
$$

Notice such a $\lambda_{s}$ must exist as $F$ is infinite. Now define $W_{s+1}=\left(W_{s} \cup\left\{z+\lambda_{s} b_{s}\right\}\right)^{*}$, and go to stage $s+2$. Otherwise, let $e$ be least such that $P_{e}$ requires attention via $x_{e}$. Set $W_{s+1}=\left(W_{s} \cup\left\{x_{e}\right\}\right)^{*}$. To complete the construction set $W=\bigcup_{s}\left(W_{s}\right)$.

End of construction.

It is easy to prove by induction that $\operatorname{dim}(V / W)=1$ and $z \notin W$. We must verify the remaining lemmata.

LEMMA 3.4. $\lim _{s} R(e, s)=R(e)$ exists and $\Phi_{e}(W) \neq C$.

ProOF. Assume $C=\Phi_{e}(W)$, and so $\lim _{s} l(e, s)=\infty$. We say $N_{e}$ is injured at stage $s+1$, if $x \in\left(W_{s+1}\right)^{*}-\left(W_{s}\right)^{*}$, and $x$ is not $e$-good. Notice that there exists a stage $s^{\prime}$, such that $\forall s>s^{\prime}\left(N_{e}\right.$ is not injured at stage $\left.s\right)$ since injuries can only occur after stage $e$ due to the action of some $P_{e}$ for $j<e$. Now we follow the reasoning of Soare [So1] to note that if $C=\Phi_{e}(W)$ then $C$ is recursive. Similarly $\lim _{s} R(e, s)=R(e)$ exists and is finite.

LEMMA 3.5. All the $P_{e}$ are met.

ProOF. Let $e$ be least such that $P_{e}$ is not met. Then $\bar{w}_{e}=W$, and $W \equiv_{T}$ $\omega_{e} \equiv_{T} \varnothing$. Let $t$ be a stage such that $\forall s>t(R(e, s)=R(e)=R(e, t))$. As $V \not_{T} \varnothing, \operatorname{dim}(V)=\infty$. Consequently there exists a stage $t^{\prime}>t$, such that

$$
\forall s>t^{\prime} \forall y\left(y \in\left(V_{s}\right)^{*}-\left(V_{t}\right)^{*} \rightarrow y \text { is } e \text {-good }\right),
$$


namely when $V$ fixes itself on $R(e)$. We claim

$$
\exists s_{1}, s_{2}>t^{\prime}\left(s_{1} \geq s_{2} \text { and } x \in \omega_{e, s_{2}} \text { and }\left(x \in V_{s_{1}+1}^{*}-V_{s_{1}}^{*}\right)\right) .
$$

If so, the result would now follow, since at stage $s_{1}, P_{e}$ would require attention as $x$ would be $e$-good. Suppose, therefore, that the claim fails. Then

$$
\forall s>t^{\prime}\left(x \in \omega_{e, s}-\omega_{e, t^{\prime}} \rightarrow\left(x \in V_{s}^{*} \text { or } x \notin V\right)\right) .
$$

Given $y \in \omega=V_{\infty}$, find a stage $t_{y}$ such that $t_{y}>t^{\prime}$ and $\omega_{e}[y]=\omega_{e, t_{y}}[y]$. (Here we are assuming $\omega_{e}$ is recursive.) It follows that $y \in \omega_{e} \cap V$ iff $y \in \omega_{e, t_{y}} \cap\left(V_{t_{y}}\right)^{*}$. We show that this implies $V \equiv_{T} \varnothing$ contrary to hypothesis. As $\omega_{e}$ is recursive and $W=V-\omega_{e}$ is recursive, it follows that $V \equiv_{T} V \cap \omega_{e}$. But now $x \in V \cap \omega_{e}$ iff $x \in \omega_{e}$ and $x \in\left(V_{t_{x}}\right)^{*}$. This is recursive and so $V \equiv_{T} \varnothing$. Therefore all the $P_{e}$ are met.

In the above examples, it is always the case that if $W \oplus\{x\}^{*}=V$, then $d(D(W)) \vee$ $d\left(\{x\}^{*}\right)=d(D(V))$. This then leads to the hope that perhaps for dependence degrees, if $V \oplus W=Q$, then $d(D(V)) \vee d(D(W))=d(D(Q))$. Retzlaff [Rt] however, showed that there were a pair of independent decidable r.e. subspaces $D_{1}, D_{2}$ with $D_{1} \oplus D_{2}$ not decidable. Ash and Downey [AD] extended this to show (over any $F)$ :

THEOREM 3.4. Let $V \in L\left(V_{\infty}\right)$ and suppose $Q \oplus W=V$ with $Q, W \in L\left(V_{\infty}\right)$. Then if $\operatorname{dim}(W)=\infty$ there exists a decidable $Q^{\prime} \in L\left(V_{\infty}\right)$ with $Q^{\prime} \oplus W=V$. Consequently every r.e. subspace can be decomposed into the direct sum of a pair of decidable r.e. subspaces.

In a sense, this result shows that for some theorems on $L\left(V_{\infty}\right)$, new techniques must be developed. Thus, for example, an analogue of Sacks' splitting theorem due to Shore [Sh] required both Sacks' strategy of preserving agreements and a strategy of creating disagreements. This theorem is

THEOREM 3.5 (SHORE $[\mathbf{S h}])$. If $V \in L\left(V_{\infty}\right)$ is not decidable, then there exists $V_{1}, V_{2} \in L\left(V_{\infty}\right)$ such that $V_{1} \oplus V_{2}=V$ and $\left.D\left(V_{1}\right)\right|_{T} D\left(V_{2}\right)$.

There have been some other more technical results in $[$ AD, Do2, Rt, Re1, NR1,2], particularly concerning lattice-theoretic combinations with these splitting theorems, but really no stronger degree-theoretic ones. Later we shall indicate some of the more recent degree-theoretic results asserting that r.e. subspaces with certain types of splittings occur in $L\left(V_{\infty}\right)$.

Before doing so, we turn to r.e. bases. Really, the first result here is due to Dekker [De], namely

THEOREM 3.6 (DEKKER [De]). If $V \in L\left(V_{\infty}\right)$ then $V$ has a recursive basis $R$.

Proof (SKETCH). Certainly, it has an r.e. basis $B=\left\{b_{0}, b_{1}, \ldots\right\}$. Construct $R=\bigcup_{s} R_{s}$. At stage $s+1$, if $b_{s} \in\left(R_{s}\right)^{*}$ set $R_{s+1}=R_{s}$. If $b_{s} \notin\left(R_{s}\right)^{*}$ find the least $b_{j} \notin\left(R_{s}\right)^{*}$ such that $\left\{b_{s}+b_{j}, b_{j}\right\} \cup R_{s}$ is independent and both $b_{s}+b_{j}, b_{j}>s$. Set $R_{s+1}=R_{s} \cup\left\{b_{s}+b_{j}, b_{j}\right\}$. It is easy to see that $y \in R$ iff $y \in R_{y+1}$, hence $R$ is recursive. 
On the other hand, Remmel's process from [Re1] shows that

THEOREM 3.7 (REMMEL [Re1]). Let $V \in L\left(V_{\infty}\right)$. Then $V$ has an r.e. basis $R \equiv_{T} V\left(\right.$ in fact, $\left.R \equiv_{W} V\right)$.

ProOF (SKETCH). Let $B=\left\{b_{0}, b_{1}, \ldots\right\}$ be an r.e. basis of $V$. Define $R_{0}=\left\{b_{0}\right\}$ and $R_{s+1}=R_{s} \cup\left\{x_{s}\right\}$ where $x_{s}=\mu y\left(y \in\left\{b_{0}, \ldots, b_{s+1}\right\}^{*}-\left\{b_{0}, \ldots, b_{s}\right\}^{*}\right)$. It is easy to see that $(R)^{*}=(B)^{*}$ by exchange. As $R$ is an r.e. basis $R \leq_{T} V$. To see $V \leq_{T} R$ given $x$ find the least stage $s$ where $R[x+1]=R_{s}[x+1]$. It is not too difficult to see that $x \in V$ iff $x \in\left(R_{s}\right)^{*}$.

These two results have several interesting consequences. Immediately, we get

COROLLARY 3.8 (REMMEL [Re1]). Let $V$ be any r.e. nonrecursive subspace. Then $V$ has infinitely many r.e. bases of incomparable $T$-degrees.

ProOF. Apply (3.7) to get $R$, an r.e. basis of $V$ with $R \equiv_{T} V$. Take any Sacks' splitting of $R=R_{1} \sqcup R_{2}$ with $\left.R_{i}\right|_{T} R_{j}$. Apply (3.6) to get a recursive basis $D_{2}$ for $\left(R_{2}\right)^{*}$, and a recursive basis $D_{1}$ for $\left(R_{1}\right)^{*}$. Then $R_{1} \cup D_{2}$ and $R_{2} \cup D_{1}$ are r.e. bases with $\left.R_{1} \cup D_{2}\right|_{T} R_{2} \cup D_{1}$. Now use iterated Sacks' splitting.

These results lead to a very fundamental question: how does $B(V)$ lie in $[\mathbf{0}, d(V)]$ ? (Recall that $B(V)$ denotes the collection of degrees of r.e. bases of $V$.) In particular, for example, does $B(V)=\{\delta: \delta \in[\mathbf{0}, d(V)]\}$ ?

Ultimately, the solution to this particular question leads to the USP/non-USP results of Lerman and Remmel $[\mathbf{L R 1 , 2}]$ on r.e. sets and their later extensions by various authors [Do3, AS2, AF, DW]. Choosing the strongest (negative) results, we have via $[\mathbf{D W}]$,

THEOREM 3.9. (i) If $X$ and $Y$ are r.e. sets with $X<_{T} Y$, there exists $V \in L\left(V_{\infty}\right)$ such that $B(V)$ is not dense in $[0, T-\operatorname{deg}(V)]$ and $X<_{T} V<_{T} Y$.

(ii) There exists $V \in L\left(V_{\infty}\right)$ such that $B(V)$ is not dense and $V \equiv_{T} \mathbf{0}^{\prime}$.

(iii) There exists an r.e. degree $\delta$ such that if $V \in L\left(V_{\infty}\right)$ and $V$ has degree $\delta$, then $B(V)$ is not dense in $[\mathbf{0}, T-\operatorname{deg}(V)]$.

PROOF (i) (FOR EXAMPLE). Downey and Welch [DW] showed that there exists an r.e. set $A$ with $X<_{T} A<_{T} Y$ such that there exist r.e. sets $\varnothing<_{T} C<_{T} B<_{T} A$, with $C<_{W} B$ for which if $E$ is r.e. and $E \leq_{W} A$, then either $C \varliminf_{W} E$ or $E \varliminf_{W} B$. Let $V=(A)^{*}$, where we consider $A$ as an r.e. subset of a recursive basis of $V_{\infty}$. Then $A \equiv_{W} V$ and if $Q$ is an r.e. basis of $V$ then $Q \leq_{W} V$ and so $Q \leq_{W} A$.

(ii) and (iii) are similar.

We remark that these results work equally well on splittings, since they only use the fact that if $B$ is an r.e. basis of $V$ then $B \leq_{W} V$. (They also therefore apply to other effective algebra settings: boolean algebras [Re], theories, etc.)

Another recent result in this vein, is due to Downey and Stob [DS], and gives extensions of (3.9). We say $V \in L\left(V_{\infty}\right)$ has the antibasis property, if there is an r.e. set $B$ with $\varnothing<_{T} B<_{T} V$, such that whenever $R$ is an r.e. basis of $V$ with $R \leq_{T} B, R \equiv_{T} \varnothing$. (Similarly: antisplitting property.) We have

THEOREM 3.10 (DOWNEY AND STOB [DS]). (i) There exists $V \in L\left(V_{\infty}\right)$ with the antibasis and antisplitting properties.

(ii) In fact, there exists a nonzero r.e. degree $\delta$ such that if $A$ is r.e. and $d(A)=\delta$, then there exists an r.e. $B$ with $\varnothing<_{T} B \leq_{T} A$, such that for all r.e. sets $C$, if $C \leq_{T} B$ and $C \leq_{W}$ A then $C \equiv_{T} \varnothing$. 
(iii) Consequently there is a completely antibasis degree: a nonzero r.e. degree $\delta$ such that for all $V \in L\left(V_{\infty}\right)$ with $d(V)=\delta, V$ has the antibasis property.

We remark that (ii) $\rightarrow$ (iii) by the techniques of (3.9). We close this section by giving a related result concerning complementation in $V_{\infty}$ (which by [DR1] is also connected with splittings and bases), and which also uses $W$-degrees. In [Do4], Downey showed that there were very few lattice-theoretic restrictions on the complement of a fully co-r.e. (immune) subspace. However in [DR1], Downey and Remmel showed that this is not the case for degrees. As we shall now show, these results may be deduced from $[\mathbf{L R 1 , 2}]$ and some $W$-degree-theoretic results.

THEOREM 3.11. Suppose $V \in L\left(V_{\infty}\right)$ and $R$ is fully co-r.e. with $V \oplus R=V_{\infty}$. Then $V \leq_{W} D(V) \leq_{W} R$.

Proof. Let $R=(B-A)^{*}$ where $B$ is a recursive basis of $V_{\infty}$ and $A$ is an r.e. subset of $B$. Let $B=\left\{b_{1}, b_{2}, \ldots\right\}$. Let $x_{1}, \ldots, x_{n}$ be given. To decide if $\left\{x_{1}, \ldots, x_{n}\right\}^{*} \cap V=\{\overrightarrow{0}\}$ or not, compute $P=\bigcup_{i} \operatorname{supp}_{B}\left(x_{i}\right)$ where $\operatorname{supp}_{B}(x)$ denotes the support of $x$ over $B$. Then $P=\left\{b_{i_{0}}, b_{i_{1}}, \ldots, b_{i_{m}}\right\}$ with $i_{j}<i_{j+1}$. Now compute the least stage $s$ where $A_{s}\left[b_{i_{m}}+1\right]=A\left[b_{i_{m}}+1\right]$. Then from this we may compute $R\left[b_{i_{m}}\right]=\left\{b_{j_{0}}, \ldots, b_{j_{k}}\right\}$. Now, find the least stage $s_{1}$ where

$$
P \subset\left(\left(V_{s_{1}}\right)^{*} \oplus\left(\left\{b_{j_{0}}, \ldots, b_{j_{k}}\right\}\right)^{*}\right)\left[b_{i_{m}}\right] .
$$

We claim $\{\overrightarrow{0}\}=\left\{x_{i}, \ldots, x_{n}\right\}^{*} \cap V$ iff $\left\{x_{1}, \ldots, x_{n}\right\}^{*} \cap\left(V_{s_{1}}\right)^{*}=\{\overrightarrow{0}\}$. If not, some linear combination of $x_{1}, \ldots, x_{n}$ and so of $b_{i_{1}}, \ldots, b_{i_{m}}$ must enter $V$ after stage $s_{1}$. But this will force $\left\{b_{j_{0}}, \ldots, b_{j_{k}}\right\}$ to be no longer independent over $V$, giving a contradiction.

Finally, we would like to mention one result due to Downey and Remmel, which will appear elsewhere. Downey [Do4] has shown that any nonzero r.e. degree contains an r.e. set without the universal splitting property. For vector spaces however, Downey and Remmel have (cf. [DR3]) shown that every r.e. degree with the universal weak truth table reduction property (cf. [LR1]) is completely UBP. That is, every subspace of such degree has the universal basis property. These results will appear elsewhere.

4. Splitting theorems. The results from the latter half of $\S 3$ seem to indicate that the degrees of splittings, bases and particularly dependence degrees of splittings, seem to be fairly unrelated, so that perhaps the results of Theorems 3.6 and 3.7 may be the best possible. The following result is therefore quite surprising.

THEOREM 4.1. Let $V \in L\left(V_{\infty}\right)$. Then for any $T$-degree $\delta$

(i) $\delta$ is the degree of an r.e. basis of $V$ iff

(ii) $\delta$ is the degree of an r.e. direct summand of $V$ iff

(iii) there exist $W, Q \in L\left(V_{\infty}\right)$ with $W \oplus Q=V$ and $W \equiv_{T} D(W) \equiv_{T} \delta$.

Proof. Evidently (iii) $\rightarrow$ (ii). To see that (ii) $\rightarrow$ (i), let $V=W \oplus Q$ with $W, Q \in L\left(V_{\infty}\right)$ and $W \equiv_{T} \delta$. Then $Q$ has a recursive basis $B_{1}$ by (3.6) and by (3.7), $W$ has an r.e. basis $B_{2}$ with $B_{2} \equiv_{T} \delta$. Then $B_{1} \cup B_{2}$ is an r.e. basis of $V$ of degree $\delta$.

Finally we show (i) $\rightarrow$ (iii). We many assume $\delta \neq \mathbf{0}$, since otherwise the result follows immediately (taking $\operatorname{dim}(W)=1$ ). Let $B$ be an r.e. basis of $V$ of degree 
$\delta$. Let $R$ be an infinite recursive subset of $B$. Set $C=B-R$ so that $C$ is an r.e. independent set of degree $\delta$.

Our construction is performed in two stages. First we construct a sequence of pairwise disjoint finite sets $\left\{F_{x}\right\}_{x \in \omega}$ contained in $R$ such that $\bigcup_{x \in \omega}\left\{x+r \mid r \in F_{x}\right\}$ is an independent set, and for each $x, \operatorname{card}\left(F_{x}\right)=x+1$. We construct the $F_{x}$ 's in stages. First, fix some enumeration of all finite subsets of $V_{\infty},\left\{D_{x}\right\}_{x \in \omega}$, so that $\operatorname{dim}\left(\left(\bigcup_{y \leq x} D_{y}\right)^{*}\right) \leq x$. Let $R=\left\{r_{0}<r_{1}<\cdots\right\}$ and suppose $\overrightarrow{0}$ has Gödel number 0 .

Stage 0. Let $F_{0}=\left\{r_{0}\right\}$.

Stage $s+1$. Assume we have defined $F_{0}, \ldots, F_{s}$ such that for each $i, F_{i} \subseteq R$ and $\operatorname{card}\left(F_{i}\right)=i+1$ and $A_{s}=\bigcup_{y=0}^{s}\left\{y+r \mid r \in F_{y}\right\}$ is independent. Since $\left(A_{s} \cup\{s+1\}\right)^{*}$ is finite dimensional, and $(R)^{*}$ is not, we can effectively find the least $t$ such that

(a) $\left\{r_{t}, \ldots, r_{t+s+1}\right\} \cap\left(\bigcup_{y=0}^{s} F_{y}\right)=\varnothing$, and

(b) $\left\{r_{t}, \ldots, r_{t+s+1}\right\}$ is independent over $\left(A_{s} \cup\{s+1\}\right)^{*}$.

We now set $F_{s+1}=\left\{r_{t}, \ldots, r_{t+s+1}\right\}$. Note that because of (b), $\left\{r_{t}+(s+1), \ldots\right.$, $\left.r_{t+s+1}+(s+1)\right\}$ is independent over $A_{s}$ so that $\bigcup_{y=0}^{s+1}\left\{y+r \mid r \in F_{y}\right\}$ is independent.

Next, let $f$ be a 1-1 recursive function whose range is $C$. We construct the desired r.e. subspace $W$ in stages as follows:

Stage 0. $W_{0}=\left(\left\{r_{0}+f(0)\right\}\right)^{*}$ where $r_{0}=\mu r\left(r \in F_{f(0)}\right)$.

Stage $s+1$. Having defined $W_{s}=\left(\left\{r_{i}+f(i) \mid i=0, \ldots, s \text { and } r_{i} \in F_{f(i)}\right\}\right)^{*}$, for some $r_{0}, \ldots, r_{s}$ we let $r_{s+1}$ be the least $r$ such that

(a) $r \in F_{f(s+1)}$,

(b) $\left(W_{s} \cup\{r+f(s+1)\}\right)^{*} \cap\left(\bigcup_{y_{\leq}(s+1)} D_{y}\right)^{*}=\left(W_{s}\right)^{*} \cap\left(\bigcup_{y_{\leq}(s+1)} D_{y}\right)^{*}$.

The point here is that such an $r$ must exist since otherwise if $F_{f(s+1)}=\left\{x_{1}, \ldots\right.$, $\left.x_{f(s+1)+1}\right\}$ then for all $i, q_{i}+x_{i} \in\left(\bigcup_{y_{\leq}(s+1)} D_{y}\right)^{*}$ for some $q_{i} \in\left(W_{s}\right)^{*}$. By our construction of the $F_{y}$ 's, it is easy to see that $q_{1}+x_{1}, \ldots, q_{f(s+1)+1}+x_{f(s+1)+1}$ are independent, which would imply that $\operatorname{dim}\left(\bigcup_{y \leq f(s+1)} D_{y}\right)^{*}>f(s+1)$; a contradiction since $\operatorname{dim}\left(\left(\bigcup_{y \leq x} D_{y}\right)^{*}\right) \leq x$ for all $x$.

Now set $W_{s+1}=\left(W_{s} \cup\left\{r_{s+1}+f(s+1)\right\}\right)^{*}$ and $W=\bigcup_{s} W_{s}$.

It is easy to see that $W \oplus(R)^{*}=V$ since $f(s) \in W \oplus(R)^{*}$ for all $s$ :

First $f(s) \in W+(R)^{*}$ for all $s$ since $r+f(s) \in W$ for some $r \in R$ (namely $\left.r=r_{s+1}\right)$. Also $W \cap(R)^{*}=\{\overrightarrow{0}\}$ because $f(s)$ exchanges with $r_{s}+f(s)$ over $(R)^{*}$ and so $\left\{r_{i}+f(i): i \in \omega\right\}$ is independent over $(R)^{*}$ since $C=\{f(i): i \in \omega\}$ is independent over $(R)^{*}$. Thus we let $Q=(R)^{*}$ and verify that $d(W)=d(D(W))=\delta$. First note that $x \in C$ iff $r+x \in W$ some $r \in F_{x}$ so that $C \leq_{T} W$. Thus it suffices to show that $D(W) \leq_{T} C$ since $W \leq_{T} D(W)$ (cf. for example [MN2]).

Given an index $r$ of some $k$-tuple $\left\langle r_{1}, \ldots, r_{k}\right\rangle$, find the first $x$ such that $D_{x}=$ $\left\langle r_{1}, \ldots, r_{k}\right\rangle$ and then recursively in $C$ find a stage $s_{x}$ such that $f(t)>x$ for all $t \geq s_{x}$. We claim $r \in D(W)$ iff $\left\langle r_{1}, \ldots, r_{k}\right\rangle$ is dependent over $W$ iff $\left(D_{x}\right)^{*} \cap W \neq(\varnothing)^{*}$ iff $\left(D_{x}\right)^{*} \cap\left(W_{s_{x}}\right)^{*} \neq\{\overrightarrow{0}\}$. That is, if $\left(D_{x}\right)^{*} \cap\left(W_{s}\right)^{*}=\{\overrightarrow{0}\}$ but $\left(D_{x}\right)^{*} \cap\left(W_{s+1}\right)^{*} \neq$ $\{\overrightarrow{0}\}$; then clearly $f(s+1)<x$ since by construction $\left(W_{s}\right)^{*} \cap\left(\bigcup_{y \leq f(s+1)} D_{y}\right)^{*}=$ $\left(W_{s+1}\right)^{*} \cap\left(\bigcup_{y \leq f(s+1)} D_{y}\right)^{*}$ and hence $s+1 \leq s_{x}$. Thus $D(W) \leq_{T} C$ and so $C \leq_{T} W \leq_{T} D(W) \leq_{T} C$ and result. 
COROllary 4.2. Let $V \in L\left(V_{\infty}\right)$. Then for any $W$-degree $\delta, \delta$ is the degree of an r.e. basis of $V$ iff there exist $W, Q \in L\left(V_{\infty}\right)$ with $W \oplus Q=V$ and $Q \equiv_{W}$ $D(Q) \equiv_{W} \delta$.

ProOF. All the reductions of the previous theorem are $W$-reductions.

The construction of Theorem 4.1 allows us to prove the following result, which says that pairs of $T$ - or $W$-degrees arising from r.e. splittings of r.e. bases of $V$ coincide exactly with degrees of strong r.e. splittings of $V$ itself.

THEOREM 4.3. For any degrees $(T$ - or $W-) \gamma$ and $\delta$ and $V \in L\left(V_{\infty}\right)$, there exists an r.e. splitting $B_{1} \cup B_{2}=B$ of an r.e. basis $B$ of $V$ with $d\left(B_{1}\right)=\gamma$ and $d\left(B_{2}\right)=\delta$ iff there exists an r.e. splitting $W_{1} \oplus W_{2}=V$ of $V$ with

(a) $d\left(W_{1}\right)=d\left(D\left(W_{1}\right)\right)=\gamma$, and

(b) $d\left(W_{2}\right)=d\left(D\left(W_{2}\right)\right)=\delta$.

Proof. We may assume $\operatorname{dim}(V)=\infty$ lest the theorem is trivially satisfied. First assume we are given disjoint r.e. sets $B_{1} \cup B_{2}=B$ an r.e. basis of $V$ and with no loss of generality both $B_{1}$ and $B_{2}$ are infinite. Observe that in the proof of Theorem 4.1, although $R$ is recursive, the only properties used in the construction of $W$ are that $R$ and $C$ are infinite r.e. sets, $R \cap C=\varnothing$ and $R \cup C$ is a basis of $V$. Thus apply the construction of Theorem 4.1 with $B_{1}$ replacing $C$ and $B_{2}$ replacing $R$ to construct $W_{1}$ so that $W_{1} \oplus\left(B_{2}\right)^{*}=V$ and $d\left(W_{1}\right)=d\left(D\left(W_{1}\right)\right)=\gamma=d\left(B_{1}\right)$. Next apply the construction of Theorem 4.1 with $B_{2}$ replacing $C$ and $R$ replaced by any r.e. basis of $W_{1}$ to construct $W_{2}$ with $W_{2} \oplus W_{1}=V$ and $d\left(W_{2}\right)=d\left(D\left(W_{2}\right)\right)=d\left(B_{2}\right)$.

For the reverse direction, apply Remmel's construction from [Re1] to produce $B_{1} \equiv_{W} W_{1}$ and $B_{2} \equiv_{W} W_{2}$.

Theorem 4.3 has a number of interesting corollaries. For example if we start with any $V \in L\left(V_{\infty}\right)$ and any recursive basis $B$ of $V$ and $B_{1} \cup B_{2}=B$ any r.e. splitting of $B$ we get Corollary 4.4 .

COROllary 4.4 (ASH AND Downey). For any $V \in L\left(V_{\infty}\right)$, there exist decidable subspaces $D_{1}$ and $D_{2}$ such that $D_{1} \oplus D_{2}=V$.

Another example is the following extension of [AD].

COROLlaRY 4.5. Let $V \in L\left(V_{\infty}\right)$ and suppose $W, Q \in L\left(V_{\infty}\right)$ with $W \oplus Q=V$ and $\operatorname{dim}(W)=\infty$. Let $H$ be any r.e. basis of $Q$. There exists $R \in L\left(V_{\infty}\right)$ with $d(R)=d(D(R))=d(H)$ such that $W \oplus R=V$.

As a final example, we prove the following classification theorem, solving a question of Ash and Downey [AD]. Following [AD] we say $V \in L\left(V_{\infty}\right)$ is everywhere mitotic if for all r.e. degrees $\delta \leq_{T} d(V)$ there exist $V_{1}, V_{2} \in L\left(V_{\infty}\right)$ such that $d\left(V_{1}\right)=d\left(V_{2}\right)=\delta$ and $V_{1} \oplus V_{2}=V$ and say $V$ is strongly everywhere mitotic if the conclusion also holds with $d\left(D\left(V_{1}\right)\right)=d\left(D\left(V_{2}\right)\right)=\delta$. We have

COROllary 4.6. Let $\delta$ be an r.e. degree. Then

(i) $\delta$ contains an r.e. set with the universal splitting property, iff

(ii) $\delta$ contains an r.e. subspace with the universal basis propery, iff

(iii) $\delta$ contains an r.e. subspace that is everywhere mitotic, iff

(iv) $\delta$ contains an r.e. subspace that is strongly everywhere mitotic, iff 
(v) $\delta$ contains an r.e. subspace $V$ such that if $\alpha$ and $\beta$ are r.e. degrees with $\alpha, \beta \leq_{T} V$, there exists $V_{1}, V_{2} \in L\left(V_{\infty}\right)$ such that $V_{1} \oplus V_{2}=V$ and $d\left(V_{1}\right)=$ $d\left(D\left(V_{1}\right)\right)=\alpha$ and $d\left(V_{2}\right)=d\left(D\left(V_{2}\right)\right)=\beta$.

Proof. Clearly (iv) $\rightarrow$ (iii) $\rightarrow$ (ii) $\rightarrow$ (i). To show (i) $\rightarrow$ (iv) let $A_{1}, A_{2}$ be r.e. subsets of a recursive basis with $A_{1}, A_{2}$ both having USP (universal splitting property), and $A_{1} \equiv_{T} A_{2} \equiv_{T} \delta$. Let $\gamma \leq_{T} \delta$. As the $A_{i}$ have U.S.P. there exist r.e. sets $B_{1}, B_{2}, C_{1}$ and $C_{2}$ such that $B_{1} \equiv_{T} B_{2} \equiv_{T} \gamma$ and $B_{i} \sqcup C_{i}=A_{i}$ for $i=1,2$. Now $\left(B_{1}\right)^{*} \oplus\left(C_{1}\right)^{*} \oplus\left(B_{2}\right)^{*} \oplus\left(C_{2}\right)^{*}=V$. Let $F_{i}$ be a recursive basis of $C_{i}$ and observe that $B_{i} \cup F_{i}$ is an r.e. basis of $\left(B_{i}\right)^{*} \oplus\left(C_{i}\right)^{*}$ for $i=1,2$. Now apply Corollary 4.5 twice to get (iv).

Finally it is clear (v) $\rightarrow$ (i). To get (i) $\rightarrow$ (v) use the proof that (i) $\rightarrow$ (iv) taking $B_{1} \equiv_{T} \alpha$ and $B_{2} \equiv_{T} \beta$.

Another interesting result which may be derived from Theorem 4.3 is a complete analogue of Sack's splitting theorem (e.g. [So1]) for both degrees and dependence degrees. Recall that Shore [Sh] proved an analogue of the Sacks' splitting theorem for dependence degrees, that is, Shore proved that if $V \in L\left(V_{\infty}\right)$ and $D(V) \not \equiv_{T} \mathbf{0}$, then there exist $V_{1}, V_{2} \in L\left(V_{\infty}\right)$ such that $V_{1} \oplus V_{2}=V$ and $\left.D\left(V_{1}\right)\right|_{T} D\left(V_{2}\right)$, although no claim about the degrees of $V_{1}$ and $V_{2}$ is made (3.5). In the case where the space $V$ is recursive but not decidable, Shore's result is the best possible since in that case $V_{1} \equiv_{T} V_{2} \equiv_{T} \mathbf{0}$. (Recursive, but not decidable, r.e. subspaces may exist only if $F$ is infinite.) However if $d(V)$ is nonzero, we can obtain the following strengthening of Shore's result.

THEOREM 4.7. (a) Suppose $V$ is an r.e. nonrecursive subspace of $V_{\infty}$. Then there exist $W_{1}$ and $W_{2} \in L\left(V_{\infty}\right)$ such that

(i) $W_{1} \oplus W_{2}=V$,

(ii) $d\left(W_{1}\right)=d\left(D\left(W_{1}\right)\right)$ and $d\left(W_{2}\right)=d\left(D\left(W_{2}\right)\right)$ and

(iii) $\left.d\left(W_{1}\right)\right|_{T} d\left(W_{2}\right)$ and $\left.d\left(D\left(W_{2}\right)\right)\right|_{T} d\left(D\left(W_{2}\right)\right)$.

(b) Moreover, given any pair $W_{1}, W_{2}$ as above there exists an infinite collection $Q_{i}$ such that $W_{1} \oplus Q_{i}=V$ and (ii) and (iii) hold in the above with $Q_{i}$ in place of $W_{2}$ such that for all $i \neq j,\left.Q_{i}\right|_{T} Q_{j}$.

ProOF. For (a) let $B$ be a recursive basis of $V$ such that $B \equiv_{T} V$. Sacks' split $B=B_{1} \cup B_{2}$, that is assume $B_{1}, B_{2}$ are r.e. disjoint sets with $\left.B_{1}\right|_{T} B_{2}$. Now apply Theorem 4.3. For (b) Sacks' split $B_{2}$ into infinitely many pairwise Turing incomparable pieces $B_{2}=\bigcup_{i} C_{i}$, each of which is Turing incomparable with $B_{1}$ (cf. $\left[\right.$ So1,2,3]). By Theorem 4.1, each $C_{i}$ has the same degree as a basis of $\left(B_{2}\right)^{*}$. Now apply Corollary 4.5.

In the next section we shall show that these results are in a sense the best possible. Before doing this, however, we would like to prove one related result for non-r.e. bases.

THEOREM 4.8. (i) Suppose $V \in L\left(V_{\infty}\right)$ is infinite dimensional, any $\delta$ is any degree. Then $V$ has a basis of degree $\delta$.

(ii) Consequently, if $\alpha_{1}$ and $\alpha_{2}$ are any degrees, there exist $V_{1}, V_{2}$ subspaces of $V$, with $V_{1} \oplus V_{2}=V$ and $D\left(V_{i}\right) \equiv_{T} V_{i} \equiv_{T} \alpha_{i}$ for $i=1,2$. 
ProOF. (i) Let $A$ be a set of degree $\delta$ and $B$ a recursive basis of $V$ with $B=\left\{b_{0}<b_{1}<\cdots\right\}$. Define $Q=\left\{x_{0}<x_{1}<\cdots\right\}$ via

$$
x_{i}= \begin{cases}b_{i} & \text { if } i \notin A \text { or } i=0, \\ b_{0}+b_{i} & \text { if } i \in A .\end{cases}
$$

It is easy to see that $Q \equiv_{T} A$ (in fact $Q \equiv_{W} A$ ).

(ii) Split $V$ into a pair of r.e. subspaces $W_{1} \oplus W_{2}=V$.

Applying (i) we get basis $Q_{1}, Q_{2}$ of $W_{1}, W_{2}$ respectively with $d\left(Q_{i}\right)=\alpha_{i}$. Now apply an oracle version of the reasoning of 4.3 to give the desired result. We leave the details to the reader.

5. The basis reflection property. The results of the last section show that the degrees of bases correspond to the degrees of decompositions in a very strong way. This leads us to hope for even stronger results. One of the most natural hopes/conjectures (which would considerably simplify many existence results) is the following:

(5.1) Let $A$ be an r.e. subset of a recursive basis of $V_{\infty}$, and let $Q$ be an r.e. basis of $(A)^{*}$. Then there is an r.e. splitting $A_{1} \sqcup A_{2}=A$ of $A$ with $A_{1} \equiv_{T} Q$.

In view of the results of $\S 3,(5.1)$ would also assert that if $W$ is an r.e. summand of $(A)^{*}$, then there exists $A_{1}$ with $W \equiv_{T} A_{1}$ as above. (5.1) would immediately reduce many questions concerning $B(V)$ and $S(V)$ to questions about r.e. sets and their splittings. Thus, for example, a non-USP r.e. subset of a recursive basis of $V_{\infty}$ would also be non-UBP.

In view of these many nice consequences, it is unfortunate that (as we shall now prove), (5.1) fails. Define $(A)^{*}$ to have basis reflection property (BRP) if it satisfies (5.1).

THEOREM 5.2. Let $\delta$ be any nonzero r.e. degree. Then $\delta$ contains an r.e. subspace without BRP.

ProOF. Fix $B=\left\{a_{0}<a_{1}<\cdots\right\}$ as a recursive basis of $V_{\infty}$. We build $A \subseteq B$ with $A=\bigcup_{s} A_{s}$ and $d(A)=\delta$; and an r.e. basis $Q=\bigcup_{s} Q_{s}$ of $(A)^{*}$ stages. At each stage $s, B-A_{s}=\left\{b_{0, s}<b_{1, s}<\cdots\right\}$. Let $f$ be a 1-1 recursive function with $d(f(\omega))=\delta$. We ensure that $f(\omega) \equiv_{T} A$, by permitting and coding (on the $b_{i, s}$ ). We satisfy

$R_{e}$ : One of the following fails.

(i) $M_{e} \sqcup N_{e}=A$,

(ii) $\Gamma_{e}(Q)=M_{e}$

(iii) $\Phi_{e}\left(M_{e}\right)=Q$,

where $\left\langle M_{e}, N_{e}, \Gamma_{e}, \Phi_{e}\right\rangle$ denotes a standard enumeration of quadruples consisting of pairs of disjoint r.e. subsets of $B$ and pairs of reductions. Some of the ideas are from Downey [Do4], although the construction is much more delicate than [Do4].

Define $l(e, s)$ by

$$
\begin{aligned}
l(e, s)=\max \left\{x: \forall y<x\left(\left(M_{e, s} \sqcup N_{e, s}\right)[y]\right.\right. & =A_{s}[y] \& \\
\Gamma_{e, s}\left(Q_{s} ; y\right) & \left.\left.=M_{e, s}(y) \& \Phi_{e, s}\left(M_{e, s} ; y\right)=Q_{s}(y)\right)\right\} .
\end{aligned}
$$

Associated with $R_{e}$ will be a restraint $r(e, s)$ restraining $Q$. We shall say that $R_{e}$ is satisfied at stage $s+1$ if for some $y$, we have either

(i) $\Gamma_{e, s}\left(Q_{s} ; y\right) \downarrow$ and $\Gamma_{e, s}\left(Q_{s} ; y\right) \neq M_{e, s}(y)$ and $u\left(\Gamma_{e, s}\left(Q_{s} ; y\right)\right)<r(e, s)$, or 
(ii) $\Phi_{e, s}\left(M_{e, s} ; y\right) \downarrow$ and $\Phi_{e, s}\left(M_{e, s} ; y\right) \neq Q_{s}(y)$ and if $u=u\left(\Phi_{e, s}\left(M_{e, s} ; y\right)\right)$ then for all $z \leq u \Gamma_{e, s}\left(Q_{s} ; z\right) \downarrow$ and $r(e, s)>u\left(\Gamma_{e, s}\left(Q_{s} ; z\right)\right.$ ). (That is, we are currently preserving a disagreement.)

Now at each stage $s$, position $\langle e, n\rangle$ of $B-A_{s}$ for each $n$ is associated with $R_{e}$ and may, or may not be assigned. We shall suppose that for all $m, n$, if $m>n,\langle e, m\rangle>$ $\langle e, n\rangle$. Now, at each stage $s$ there will be at least one element added to $A_{s+1}-A_{s}$. This will mean then at stage $s$ there is at least one linear combination $x(\langle e, n\rangle, s)$ of elements of $A_{s}$ and $B_{\langle e, n\rangle, s}$ with $x(\langle e, n\rangle, s)=\sum_{a_{i} \in A_{s}} \lambda_{i} a_{i}+\gamma b_{\langle e, n\rangle, s}(\gamma \neq 0)$, with $x(\langle e, n\rangle, s)>s$. This observation will be the key to preserving computations. In the construction to follow, action by higher priority requirements automatically cancels lower priority ones. Also, for two assigned positions $\langle e, n\rangle\langle\langle e, m\rangle$, if $\langle e, n\rangle$ requires attention then $\langle e, m\rangle$ automatically becomes unassigned.

At this stage we will briefly describe the idea for satisfying the $R_{e}$. Roughly speaking we shall proceed as follows: wait for a stage $s$ to occur where we have a 'target' $t(\langle e, n\rangle, s)$ (an element of $B$ ) and $l(e, s)>t(\langle e, n\rangle, s)$. We then assign a linear combination of $x=x(\langle e, n\rangle, s)$ as above as a follower of $R_{e}$ targeted for $Q$. Now, notice that

$$
x(\langle e, n\rangle, s)>s>\max \left\{u\left(\Phi_{e, s}\left(Q_{s} ; y\right)\right): y \leq t=t(\langle e, n\rangle, s)\right\} .
$$

We now again wait till $l(e, s)>x$. Our idea is to preserve all computations now, and wait till we can clear all elements $y$ with $t \leq y \leq s$ into $A$ and $l(e, s)>x$ again. At such a stage $x$ become confirmed. The point is that if we ever get to add $x$ to $Q$ then by restraints either the $\Phi_{e, s}\left(M_{e, s} ; x\right)$ computation is final, in which case we have disagreement $\Phi_{e, s}\left(M_{e, s} ; x\right)=0 \neq 1=Q_{e}(x)$, or some number $<u\left(\Phi_{e, s}\left(M_{e, s} ; x\right)\right)$ enters $M_{e}$. The only remaining such numbers are $<t$ and so this gives a disagreement below $t$ by choice of $x$. Formal details now follow.

We say $R_{e}$ requires attention at stage $s+1$ via $\langle e, n\rangle$ if it is unsatisfied at stage $s$, all positions $\langle e, m\rangle$ with $m<n$ are assigned or bogus, and

(5.3) $\langle e, n\rangle$ is waiting and confirmed and $b_{f(s), s} \leq t(\langle e, n\rangle, s)$, or

(5.4) $\langle e, n\rangle$ is not confirmed and $f(s) \leq\langle e, n\rangle$, or

(5.5) $\langle e, n\rangle$ is unassigned and

(i) $f(s) \leq\langle e, n\rangle$,

(ii) $\forall y \leq b_{\langle e, n\rangle, s}\left(\Gamma_{e, s}\left(Q_{s} ; y\right) \downarrow\right)$,

(iii) for some linear combination $x(\langle e, n\rangle, s)=\sum_{a_{i} \in A_{s}} \lambda_{i} a_{i}+\gamma b_{\langle e, n\rangle, s}$ of $A_{s} \cup$ $\left\{b_{\langle e, n\rangle, s}\right\}$ with $\gamma \neq 0$, we have $x(\langle e, n\rangle, s)>\max \left\{u\left(\Gamma_{e, s}\left(Q_{s} ; y\right)\right): y \leq b_{\langle e, n\rangle, s}\right\}$,

(iv) $\forall y \leq x(\langle e, n\rangle, s)\left(\Phi_{e, s}\left(M_{e, s} ; y\right) \downarrow\right)$, and

(v) if $m=\max \left\{u\left(\Phi_{e, s}\left(M_{e, s} ; y\right): y \leq x(\langle e, n\rangle, s)\right)\right\}$ then $l(e, s)>m$, and $l(e, s)>$ $R(e, s)=\max \{r(j, s): j \leq e\}$.

If (5.5) holds, we shall say that $R_{e}$ requires attention via $\langle e, n\rangle$ through $x=$ $x(\langle e, n\rangle, s)$.

Construction. Stage $s+1$.

Step 1. For all $e \leq s$ and positions $\langle e, n\rangle$ with follower $x=x(\langle e, n\rangle, s)$ if

(i) $\langle e, n\rangle$ is not waiting,

(ii) $\langle e, n\rangle$ is not confirmed, and

(iii) $l(e, s)>R(e, s)$,

declare $x$ as waiting. 
Step 2. Determine any threatened requirement as follows: see if $b_{f(s), s}=$ $t(\langle e, n\rangle, s)$ for some $\langle e, n\rangle$ which is assigned but does not require attention at stage $s+1$. (It will follow that $\langle e, n\rangle$ must be confirmed but not waiting.) In this case set $g(s)=f(s)+1$. Otherwise set $g(s)=f(s)$. (By cancellation $b_{f(s)+1, s}$ may be threatened also, but will be of lower priority.)

Step 3 . Find the least $e$ if any, such that $R_{e}$ requires attention.

(a) If no such $e$ exists, find the least $k$ such that both $b_{k, s}$ and $b_{g(s), s}+b_{k, s}$ exceed all uses, numbers, etc., used by this stage.

Set $A_{s+1}=A_{s} \cup\left\{b_{g(s), s}, b_{k, s}\right\}$. Set $Q_{s+1}=Q_{s} \cup\left\{b_{k, s}, b_{g(s), s}+b_{k, s}\right\}$. For all assigned positions $h$ with $h \geq g(s)$, cancel the assignments so that $x(h, s+1)$ and $t(h, s)$ become undefined.

$$
\text { Set } b_{i, s+1}= \begin{cases}b_{i, s} & \text { for } i<g(s), \\ b_{i+1, s} & \text { for } g(s) \leq i<k, \\ b_{i+2, s} & \text { otherwise. }\end{cases}
$$

For all $q$ set $r(q, s+1)=r(q, s)$. Otherwise do nothing.

(b) If for some $e, R_{e}$ requires attention, find the least $e$ and the corresponding $\langle e, n\rangle$. We pick $\langle e, n\rangle$ by selecting which option of (5.3)-(5.5) first holds (the highest priority option), where we give (5.3) higher priority than (5.4) which in turn is higher than (5.5). All lower priority positions (that is, $\langle j, k\rangle$ with $j>e$ ) with $\langle j, k\rangle \leq\langle e, n\rangle$ become bogus at this stage. Now adopt the appropriate case below.

Case 1. (5.3) holds. $R_{e}$ now becomes satisfied.

Subcase (i). $b_{g(s), s} \neq t(\langle e, n\rangle, s)$. Find $k$ as in (a) and set

$$
A_{s+1}=A_{s} \cup\left\{b_{g(s), s}, b_{k, s}, t(\langle e, n\rangle, s)\right\}
$$

and set $Q_{s+1}=Q_{s} \cup\left\{b_{g(s), s}+b_{k, s}, b_{k, s}, x(\langle e, n\rangle, s)\right\}$. Cancel all assignments for $h \geq g(s)$. Otherwise do nothing.

Subcase (ii). $b_{g(s), s}=t(\langle e, n\rangle, s)$. Set $A_{s+1}=A_{s} \cup\{t(\langle e, n\rangle, s)\}$ and $Q_{s+1}=$ $Q_{s} \cup\{x(\langle e, n\rangle, s)\}$. Cancel as before.

Case 2. (5.4) holds. $\langle e, n\rangle$ becomes confirmed (but not waiting).

Subcase (i). $g(s)=\langle e, n\rangle$. For each $i$ with $1 \leq i \leq s$, find $k(i)$ as in (a), so that $b_{g(s)+i, s}+b_{k(i), s}$ exceeds all previously considered numbers, and set $A_{s+1}=A_{s} \cup$ $\left\{b_{g(s)+i, s}, b_{k(i), s}: 1 \leq i \leq s\right\}$, and $Q_{s+1}=Q_{s} \cup\left\{b_{k(i), s}, b_{g(s)+i, s}+b_{k(i), s}: 1 \leq i \leq s\right\}$. Cancel as before for $h \geq g(s)+1$. For all $q \leq\langle e, n\rangle, t(q, s+1)=t(q, s)$, (if defined). Restraints are maintained. Renumber the $b_{i, s}$.

Subcase (ii). $g(s) \neq\langle e, n\rangle$. Find $i$ with $1 \leq i \leq s$ as in subcase (i), with this time $b_{\langle e, n\rangle+1}+b_{k(i), s}$ and $b_{k(i), s}$ appropriately large. Also find $k$ for $g(s)$ as in (a). Set

$$
A_{s+1}=A_{s} \cup\left\{b_{g(s), s}, b_{k, s}, b_{\langle e, n\rangle+i, s}+b_{k(i), s}: 1 \leq i \leq s\right\} .
$$

Set $Q_{s+1}=Q_{s} \cup\left\{b_{g(s), s}+b_{k, s}, b_{k, s}, b_{\langle e, n\rangle+i, s}+b_{k(i), s^{\prime}} b_{k(i), s}: 1 \leq i \leq s\right\}$. Cancel as before for $h \geq g(s)$ with $h \neq\langle e, n\rangle$. For $q \leq\langle e, n\rangle, t(q, s+1)=t(q, s)$ (if defined). (But notice here that $t(\langle e, n\rangle, s+1)=b_{\langle e, n\rangle-1, s+1}$ after renumbering the $b_{j, s}$.)

Case 3. (5.5) holds. $\langle e, n\rangle$ now becomes assigned, and $x(\langle e, n\rangle, s)$ becomes the current follower associated with $\langle e, n\rangle$.

Set $r(e, s+1)=s+1$, and $t(\langle e, n\rangle, s+1)=b_{\langle e, n\rangle, s} .\langle e, n\rangle$ is not confirmed. Find $k$ as in (a), and set $A_{s+1}=A_{s} \cup\left\{b_{g(s), s}, b_{k, s}\right\}$, and $Q_{s+1}=Q_{s} \cup\left\{b_{g(s)}+b_{k, s}, b_{k, s}\right\}$.

End of construction. 
Clearly $(Q)^{*}=(A)^{*}$ and $\lim _{s} b_{j, s}=b_{i}$ exists, since $b_{i, s+1} \neq b_{i, s}$ only if $i \geq f(s)$. This also means $A \leq_{T} f(\omega)$, since, to determine if $a_{j} \in B$ is in $A$ or not (as we know $\left.\forall s\left(b_{j, s} \geq a_{j}\right)\right)$, it suffices to find a stage $s_{0}$ where $\forall s>s_{0}(f(s)>j)$. Then $a_{j} \in A \leftrightarrow a_{j} \in A_{s_{0}}$. Also $f(\omega) \leq_{T} A$, since $b_{f(s), s}, b_{f(s)+1, s}$ or $b_{f(s)+2, s}$ is always enumerated into $A_{s+1}-A_{s}$. Thus, given $j, A$-recursively find a stage $s_{0}$ where $\forall s>s_{0}, b_{k, s}=b_{k, s_{0}}$ for all $k \leq j+3$. Then $\forall s>s_{0}(f(s)>j)$. Hence $f(\omega) \leq_{T} A$.

Thus it will suffice to show that

(i) all the $R_{e}$ are met,

(ii) all the $R_{e}$ receive attention at most finitely often,

(iii) $\lim _{s} r(e, s)=r(e)$ exists.

Since the $r(e, s)$ only change when $R_{e}$ receives attention, (ii) $\rightarrow$ (iii). Thus the remainder of the verification will be devoted to (i) and (ii), and will consist of a series of lemmas under an inductive hypothesis. Let $s_{0}$ be the least stage such that

$$
\forall j<e \forall s>s_{0}\left(R_{j} \text { does not receive attention at stage } s\right) \text {. }
$$

Now suppose, by way of contradiction, that $R_{e}$ is not met, or receives attention infinitely often. By the way followers are assigned in (5.5), it is easy to see that in either case we must suppose $l(e, s) \rightarrow \infty$ and infinitely many followers are assigned to $R_{e}$ after stage $s_{0}$. By choosing $s_{0}$ least, we may suppose that $R_{e}$ has no followers at stage $s_{0}$.

LEMMA 5.6. Suppose that at some stage $s+1>s_{0}, R_{e}$ receives attention via $\langle e, n\rangle$ and (5.3) applies. Then for all $t \geq s+1, R_{e}$ is satisfied at stage $t$.

ProOF. Let $s,\langle e, n\rangle$ be as given by the hypothesis. By the construction, and choice of $s_{0}$, it follows that at some stage $s_{1}+1$ with $s_{0}<s_{1}+1<s+1, R_{e}$ received attention via $\langle e, n\rangle$ by way of (5.5). Choosing the largest such $s_{1}+1$ we know that for all stages $t$ with $s_{1}+1 \leq t<s+1$

(i) $R_{e}$ does not receive attention at stage $t$ via $\langle e, m\rangle$ with $m<n$,

(ii) $\langle e, n\rangle$ remains assigned at stage $t$.

Now as (5.5) applied at stage $s_{1}+1$, we know

$$
x\left(\langle e, n\rangle, s_{1}+1\right)>M=\max \left\{u\left(\Gamma_{e, s_{1}}\left(Q_{s_{1}} ; y\right)\right): y \leq b_{\langle e, n\rangle, s_{1}}\right\},
$$

where $l(e, s)>u\left(\Phi_{e, s_{1}}\left(M_{e, s_{1}} ; z\right)\right)$ for $z \leq x\left(\langle e, n\rangle, s_{1}+1\right)$ (and all the computations halt).

At this stage $r\left(e, s_{1}+1\right)=s_{1}+1$. By the way we define $k$, by (i) and (ii) above and since $\langle e, n\rangle$ is assigned at stage $s+1$, we see that

(iii) $Q_{s_{1}}[M]=Q_{s}[M]$, since

(iv) $Q_{s_{1}}\left[s_{1}\right]=Q_{s}\left[s_{1}\right]$.

This follows by the above, and since $\langle e, n\rangle$ is waiting at stage $s+1$.

Now, at some stage $s_{2}$ with $s_{1}+1<s_{2}+1<s+1,\langle e, n\rangle$ must have been confirmed via (5.4). This means that for all $x \in B$, if $b_{\langle e, n\rangle, s_{1}}<x \leq s_{1}$, then $x \in A_{s_{2}+1}$. As $\langle e, n\rangle$ became waiting at some stage $s_{3}+1$ with $s_{2}+1<s_{3}+1 \leq s+1$, it follows that at stage $s_{3}, l\left(e, s_{3}\right)>s_{1}$. This means by (iii) and (iv) above, if $x \in A$ and $t(\langle e, n\rangle, s)=b_{\langle e, n\rangle, s_{1}}<x \leq s_{1}$ then in fact $x \in A_{s_{1}}$.

Now, at stage $s+1$ we act by putting $t(\langle e, n\rangle, s)$ into $A$ and more importantly, $x(\langle e, n\rangle, s)$ into $Q_{s+1}-Q_{s}$.

There are two cases. 
Case 1. $\forall t \geq s\left(A_{t}\left[s_{1}\right]=A\left[s_{1}\right]\right)$. Then we shall restrain forever the disagreement

(v) $\Phi_{e, t}\left(M_{e, t} ; x(\langle e, n\rangle, s)\right)=0 \neq 1=Q_{t}(x(\langle e, n\rangle, s))$.

Case 2. $\exists t^{\prime} \geq s\left(A_{t}\left[s_{1}\right] \neq A\left[s_{1}\right]\right)$. Then $t^{\prime}>s$, and for all stages $t$ with $s<t<t^{\prime}$, (v) applied. At stage $t^{\prime}$ we get a new disagreement as follows: Since $A_{t^{\prime}}\left[s_{1}\right] \neq A\left[s_{1}\right]$, some $z \leq s_{i}$ enters $A_{t^{\prime}}-A_{t^{\prime}-1}$. Since $t^{\prime}-1 \geq s$, it follows that $z \leq t(\langle e, n\rangle, s)$, by our previous analysis. But by choice of $x=x(\langle e, n\rangle, s)$, we know $x>u\left(\Gamma_{e, s_{1}}\left(Q_{s_{1}} ; z\right)\right)$ and so because of the restraints imposed, $x>u\left(\Gamma_{e, t}\left(Q_{t} ; z\right)\right)$. Hence,

$$
\forall t \geq t^{\prime}\left[\Gamma_{e, t}\left(Q_{t} ; z\right)=0 \neq 1=Q_{t^{\prime}}(z)\right]
$$

Thus $R_{e}$ becomes satisfied at stage $s+1$, and does not receive attention again.

In view of this, we may now suppose that for all $\langle e, n\rangle$ assigned after stage $s_{0}$, (5.3) does not apply to $\langle e, n\rangle$. Define a position $\langle e, n\rangle$ to be permanently waiting, if it becomes waiting at some stage $s>s_{0}$ and does not get unassigned after stage $s$.

LEMMA 5.7. There are infinitely many permanently waiting positions associated with $R_{e}$.

PROOF. First we shall show that infinitely many positions get confirmed. Suppose not. Let $n_{1}, \ldots, n_{m}$ be the set of positions which are confirmed at the last stage $s_{1}$ such that any $\langle e, k\rangle$ position gets confirmed. We may suppose

$$
\left\langle e, n_{i}\right\rangle\left\langle\left\langle e, n_{i+1}\right\rangle \quad \text { for all } 1 \leq i \leq m-1 .\right.
$$

We may also suppose that by definition of $\langle$, $\rangle$, for all $p, q>n_{i+1}$, if $p>q$ then $\langle e, q\rangle+1<\langle e, p\rangle$.

Let $m(j)=\left\langle e, n_{i+1}+j\right\rangle$ for $j \geq 1$. We shall show that $f(\omega)$ is recursive contrary to hypothesis.

Let $z \in \omega$. Find the least stage $t(z)>s_{1}$ and the least $m(j)>z$ such that $m(j)$ gets assigned at stage $t(z)$. We can do this because we know that once $m(k)$ gets assigned it cannot be cancelled by choice of $g(s)$, and the fact that once $m(k)$ is assigned $f(s) \npreceq m(k)$, for then $m(k)$ would be confirmed. Then it follows by this analysis that $\forall s>t(z)(f(s)>m(j))$. Thus $\forall s>t(z)(f(s)>z)$. This implies that $z \in f(\omega)$ iff $z \in[f(\omega)]_{t(z)}$, hence $f(\omega) \equiv_{T} \varnothing$, a contradiction.

Therefore, infinitely many positions get confirmed. Now, as $l(e, s) \rightarrow \infty$ each such confirmed position must become (permanently) waiting unless cancelled by the coding strategy kills it before it becomes waiting. But our coding strategy (step 2) specifically protects the highest priority position to be threatened. We now can argue by induction that infinitely many positions must become waiting, and this will be permanent since once they become waiting, they cannot be cancelled lest (5.3) apply.

Finally, we get the theorem as follows: we shall show that $f(\omega)$ is recursive.

Let $\langle e, n\rangle$ be the first assigned permaently waiting position, and let it become so at stage $s_{1}$. Let $m(j)=\langle e, n+j\rangle$ for $1 \leq j$. We compute if $z \in f(\omega)$ or not as follows.

Find a stage $t_{1}>s_{1}$ such that for some least $m(j)$,

(i) $z<m(j)$,

(ii) $m(j)$ is waiting at stage $t_{1}$.

Then $z \in f(\omega)$ iff $z \in[f(\omega)]_{t_{1}}$, since otherwise (5.3) would apply. Consequently $f(\omega) \equiv_{T} \varnothing$, a contradiction, giving the theorem. 
Of course, some $V \in L\left(V_{\infty}\right)$ do not have BRP. For example, let $A$ be a USP r.e. subset of a recursive basis of $V_{\infty}$; then $A$ has BRP. However, on the positive side we have a better result.

THEOREM 5.8. Let $\delta$ be any r.e. degree. Then $\delta$ contains an r.e. subspace with $B R P$.

ProOF. Let $A$ be an r.e. subset of a recursive basis of $V_{\infty}$ such that $A$ is a cylinder. It is shown by Ambos-Spies and Fejér in $[\mathbf{A F}]$, that $A$ has the following property:

Let $C \leq_{W} A$; then there exists an r.e. splitting $A_{1} \sqcup A_{2}$ of $A$ with $A_{1} \equiv_{W} C$.

Now let $R$ be an r.e. basis of $(A)^{*}$. Then $R \leq_{W}(A)^{*} \equiv_{W} A$. By the property above it follows that $R$ corresponds to a splitting of $A$.

6. Strong atomicity. The results of $\S \S 5$ and 6 show that there appear to be no simple relationships between the (degrees of) r.e. splittings of an r.e. basis of $V$ and the degrees of bases of $V$ except those of $\S 4$. Indeed, the results of $\S 3$ and $\S 5$ together pin down the principal difficulty in classifying $B(V)$ :

(6.1) Suppose $W \oplus Q=V$ for $W, Q, V \in L\left(V_{\infty}\right)$. What can be said about $d(W)$, $d(Q)$ and $d(V)$ ? Furthermore, what can be said about the collection of degrees $C(W)=\left\{d\left(Q^{\prime}\right): Q^{\prime} \in L\left(V_{\infty}\right)\right.$ and $\left.W \oplus Q^{\prime}=V\right\}$ ?

The point is, that in the r.e. set case, often we can use degree-theoretic results to prove results about the possible splittings of an r.e. set $A$. For example suppose $A$ is complete. Then by Lachlan's nondiamond theorem, we cannot split $A=A_{1} \sqcup A_{2}$ with $A_{1} \mid{ }_{T} A_{2}$ and $\inf \left\{d\left(A_{1}\right), d\left(A_{2}\right)\right\}=0$. The fact that we do not know the answers to (6.1) means that a similar process fails for $L\left(V_{\infty}\right)$. The property which is (trivially) true of r.e. sets which makes this process work, is

(6.2) If $A_{1} \sqcup A_{2}=A$ then $d\left(A_{1}\right) \vee d\left(A_{2}\right)=d(A)$.

The failure of this property to hold for $L\left(V_{\infty}\right)$, means that we need new proofs for various theorems (if true, for example [Sh]) and that it may be possible that we have virtually no control over $C(W)$ of (6.1). One example of this, is that it may be possible to always split $V=Q \oplus W$ with $\varnothing<_{T} W \leq_{T} Q \leq_{T} V$. In this section, we shall show that this is not the case (answering several questions from the literature), by constructing a strongly atomic r.e. nonrecursive subspace. Recall that $V \in L\left(V_{\infty}\right)$ is strongly atomic if, whenever $V=Q \oplus W$ then $\inf \{d(Q), d(W)\}=$ 0 .

In the next section, we shall use these spaces together with some properties of $W$-degrees to further explore (6.1). Thus we shall prove-using these conceptsthe ultimate failure of (6.2), by proving there exist $V \in L\left(V_{\infty}\right)$ and $W \in L\left(V_{\infty}\right)$ such that $W$ is a summand of $V$, but for all $Q \in L\left(V_{\infty}\right)$ with $W \oplus Q=V$, $d(W) \vee d(Q) \neq d(V)$. The results of this section may therefore be viewed also as a lemma for this later construction.

In fact, we shall prove a surprisingly strong existence theorem as follows:

THEOREM 6.3. There exists a high r.e. subset $A$ of a recursive basis $R$ such that every r.e. complement of $(R-A)^{*}$ is strongiy atomic.

ProOF. Our construction is similar to that of a high minimal pair, and is a modification of Downey and Welch [DW]. Let $R$ be a recursive basis of $V_{\infty}$. We 
construct $A=\bigcup_{s} A_{s}$ in stages so that $R-A$ has the desired properties. We identify, where appropriate $R$ with $\omega$. We let $\langle$,$\rangle denote a fixed 1-1 pairing of$ $R$ and $X^{(n)}=\{y:\langle n, y\rangle \in X\}$ the $n$th slice of $X$. Recall $\omega_{e}$ denotes the $e$ th r.e. set. Define $B=\bigcup_{s} B_{s}$ as follows: let $c(e, s)=\operatorname{card}\left(\omega_{e, s}\right)$. For each $0 \leq e \leq s$, set $B_{s+1}^{(e)}=\varnothing$ if $c(e, s)=0$ and $B_{s+1}^{(e)}=\{\langle e, 0\rangle, \ldots,\langle e, c(e, s)\rangle\}$ otherwise. Now set $B^{(e)}=\bigcup B_{s}^{(e)}$ and $B=\bigcup_{e} B^{(e)}$. Now $B^{(e)}=\omega$ iff card $\left(\omega_{e}\right)=\infty$ so that if $Y$ is a thick subset of $B$ then $Y$ is high (that is, $Y \subset B$ and $Y={ }^{*} B$ ). Thus we shall meet

$$
P_{e}: A^{(e)}={ }^{*} B^{(e)} \quad \text { and } \quad A \subset B .
$$

Recall that $I_{e}$ is the $e$ th r.e. independent set and $W_{e}$ the $e$ th r.e. space with $W_{e, s}=\left\{x \in\left(I_{e, s}\right)^{*}: x \leq m\right\}$ with $m=\max \{s, n\}$ where $n=\max \left\{y \mid y \in I_{e, s}\right\}$. We must also meet the requirements

$N_{e}$ : If $e=\langle n, j, k, m\rangle$ then if

(i) $W_{j} \cap W_{k}=\{\overrightarrow{0}\}$,

(ii) $\left(W_{j} \oplus W_{k}\right)^{*} \cap(R-A)^{*}=\{\overrightarrow{0}\}$, and,

(iii) $W_{j} \oplus W_{k} \oplus(R-A)^{*}=V_{\infty}$, then,

(iv) If $\Phi_{m}\left(I_{j}\right)=\Phi_{n}\left(I_{k}\right)=f$ and $f$ is total, then $f$ is recursive.

By the well-known remark of Posner (cf. [So2]), we may replace (iv) by (iv)': If $\Phi_{m}\left(I_{j}\right)=\Phi_{m}\left(I_{k}\right)=f$ and $f$ is total then $f$ is recursive. The basic idea of meeting the $N_{e}$ is as follows. For a single requirement $N_{e}$ we associate a certain restraint $r(e, s)$ (which depends on the use functions and length of agreement generated by (i)-(iv)' above). This restraint remains active until we reach a stage where the appropriate lengths of agreement all rise to exceed the previous one. Such a stage is called (e-)expansionary. For example at this stage we may have $\left(W_{j, s}\right)^{*} \cap\left(W_{k, s}\right)^{*}=$ $\{\overrightarrow{0}\},\left(W_{k, s} \cup W_{j, s}\right)^{*} \cap\left(R-A_{s}\right)^{*}=\{\overrightarrow{0}\}$ and the lengths of agreement between $V_{\infty}$ and $\left(W_{j, s}\right)^{*} \oplus\left(W_{k, s}\right)^{*} \oplus\left(R-A_{s}\right)^{*}$ together with those of $\Phi_{m}\left(W_{j, s}\right)$ and $\Phi_{m}\left(W_{k, s}\right)$ have both exceeded our previous lengths of agreement. At this stage we enumerate one follower $x$ of some $P_{j}$ into $A_{s}$. We then raise restraints to the use functions associated with the above computations, until the next expansionary stage. We must be very careful here as we must ensure that no stray linear combinations may enter both sides below the restraint, and as in [DR1] we must enumerate nothing into $A$ which may be dependent relative to $I_{j, s} \cup I_{k, s} \cup\left(R-A_{s}\right)$ on elements of $V_{\infty}$ below $r(e, s)$ (see the definition of $(\sigma, s)$-free in the construction). In this way we can ensure that at most one element below the restraint may enter $I_{j, s} \cup I_{k, s}$ so that knowledge that $W_{j} \oplus W_{k} \oplus(R-A)^{*}=V_{\infty}$ and $\Phi_{m}\left(I_{j}\right)=\Phi_{m}\left(I_{k}\right)$ allows us to simply wait until the computations recover and $t$ cannot change below the length. In this way we show $f$ is recursive.

The interaction of the various $N_{e}$ 's presents some problems, namely we must simultaneously play many strategies according to guesses as to the final values of $r(e, s)$, and whether or not $B^{(e)}$ is infinite (so that, $e$-expansion stages and $j$ expansion stages for $j<e$ cooperate to impose essentially finite restraint on the whole construction). This may be accomplished in various ways (cf. $[$ So2, 3]). Here we use Lachlan's binary tree of strategies. We play the $P_{e}$ on the even nodes and $N_{e}$ on the odd ones. As usual, we define a binary ordering on the nodes so that we respect restraints to the left or below the node at which we are playing. By definition of "accessible node (= play)" we give a decision procedure which ensures 
the "true path", the leftmost branch is played infinitely often, contains only correct plays and our ordering ensures all the $P_{e}$ and $N_{e}$ are met.

Let $T$ be the complete binary tree. For $\sigma, \tau \in T$, write $\sigma \subseteq \tau$ if $\sigma$ is an initial segment of $\tau$ and $\operatorname{lh}(\sigma)$ for the length of $\sigma$. We define a left partial ordering < on $T$ via $\sigma<\tau \leftrightarrow\left[(\sigma \subseteq \tau) \vee \exists \gamma \in T\left(\gamma^{\wedge} 0 \subseteq \sigma\right.\right.$ and $\left.\left.\gamma^{\wedge} 1 \subseteq \tau\right)\right]$. For each $\sigma \in T$, if $\operatorname{lh}(\sigma)=2 e$, we say $\sigma$ is even and assign $P_{e}$ to $\sigma$. We therefore write $P_{\sigma}, A^{(\sigma)}, B^{(\sigma)}$ and $R^{(\sigma)}$ for $P_{e}, A^{(e)}, B^{(e)}$ and $R^{(e)}$ respectively. If $\ln (\sigma)=2 e+1$, we say $\sigma$ is odd and assign $N_{e}$ to $\sigma$. We shall write $N_{\sigma}, I_{\sigma}, W_{\sigma}, V_{\sigma}, J_{\sigma}$ and $\Phi_{\sigma}$ for $N_{e}, I_{j}, W_{j}, W_{k}, I_{k}$ and $\Phi_{m}$ respectively.

In the construction to follow, we shall enumerate where possible, unrestrained (with priority $e$ ) elements of $B$ into $A$. To do this we define $r(\sigma, s)$, the $\sigma$-restraint at stage $s$. We ensure that if $\operatorname{lh}(\sigma)>s$ then $r(\sigma, s)=0$. Define $R(\sigma, s)=$ $\max \{r,(\tau, t): \tau \leq \sigma$ and $t \leq s\}$. Notice that $\lambda s R(\sigma, s)$ is a monotone increasing function for each $\sigma \in T$. The key restraint definition is

DEFINITION. We say $x \in V_{\infty}$ is $(\sigma, s)$-free if $\forall \tau \leq \sigma\left(x \notin\left[\left(W_{\tau, s} \cup V_{\tau, s}\right) \cup R(\sigma, s)\right]^{*}\right)$.

REMARKS. (i) Observe that if $x$ is $(\sigma, s)$-free then

$$
\forall \tau \leq \sigma\left\{\left(W_{\tau, s} \cup V_{\tau, s}\right)^{*}[R(\sigma, s)]=\left(W_{\tau, s} \cup V_{\tau, s} \cup\{x\}\right)^{*}[R(\sigma, s)]\right\} .
$$

(ii) We observe that restricting additions to $A$ to only $(\sigma, s)$-free elements will allow us to meet a single $N_{e}$. We essentially must ensure that between expansions only one element may enter $W_{\tau}$ or $V_{\tau}$. Fix $\tau$. Let $W=W_{\tau}$ and $V=V_{\tau}$. Suppose we have some existing restraint $R_{s}=R(\tau, s)$, and we wish to enumerate some $x \leq R_{s}$ into $A$ for the sake of some $P_{j}$ for $j>e$. Without loss of generality let $s$ be a gap, namely the appropriate lengths of agreement expand above $R_{s}$. Specifically

(i) $W_{s}^{*} \oplus V_{s}^{*} \oplus\left(R-A_{s}\right)^{*}\left[R_{s}\right]=V_{\infty}\left[R_{s}\right]$,

(ii) $\Phi_{\tau}\left(W_{s} ; z\right)=\Phi_{\tau}\left(V_{s} ; z\right)$ for all $z \leq y$ for some $y>R_{s}$ (with maximum use $u$, say).

At this stage we place $x \in R$ into $A_{s+1}-A_{s}$, that is remove it from $\left(R-A_{s+1}\right)^{*}$. We raise $R_{s}$ to $R_{s+1}$ generated by the $y$ and $u$ above, and only add $(\tau, s+1)$-free (at least) elements to $A_{s}$. Assuming we are successful in this restraint we claim that elements $\leq R_{s}$ may enter only one of $W_{t}$ or $V_{t}$ until the next gap. For suppose

$$
\begin{aligned}
\left\{z_{1}, \ldots, z_{n}\right\}= & \left(\left(W_{s}\right)^{*} \oplus\left(V_{s}\right)^{*} \oplus\left(R-A_{s}\right)^{*}\right)\left[R_{s}\right] \\
& -\left(\left(W_{s}\right)^{*} \oplus\left(V_{s}\right)^{*} \oplus\left(R-A_{s}-\{x\}\right)^{*}\right),
\end{aligned}
$$

then also (by (i))

$$
\left\{z_{1}, \ldots, z_{n}\right\}=V_{\infty}\left[R_{s}\right]-\left(\left(W_{s}\right)^{*} \oplus\left(V_{s}\right)^{*} \oplus\left(R-A_{s}-\{x\}\right)^{*}\right) .
$$

Then if $z$ enters $\left(\left(W_{t}\right)^{*} \cup\left(V_{t}\right)^{*}\right)$ for any $t \geq s$ for any $z \in\left\{z_{1}, \ldots, z_{n}\right\}$ then we claim that by exchange, at that stage $\left(W_{t}\right)^{*} \oplus\left(V_{t}\right)^{*} \oplus\left(R-A_{t}\right)^{*}\left[R_{s}\right]=V_{\infty}\left[R_{s}\right]$. Therefore either no $z \leq R_{s}$ enters either $W_{t}$ or $V_{t}$ or exactly one $z \leq R_{s}$ may enter one of $W_{t}$ or $V_{t}$.

Recall that we wish to construct $A \subseteq^{*} B$, with $A \subseteq B$. Thus for notational convenience we define $Q(\sigma, s)=\mu z\left(z \in R\right.$ and $z$ is $(\sigma, s)$-free). To meet the $P_{\sigma}$ we employ a "guess" of $A$ defined as follows: for each $\langle s, x\rangle, x \in A_{\sigma, s} \leftrightarrow\left(\left(x \in A_{s}\right) \vee \exists \tau\right.$ $\left[\tau\right.$ is an even node and $\tau^{\wedge} 0 \subseteq \sigma$ and $x \in R^{(\tau)}$ and $x$ is $(\sigma, s)$-free $\left.]\right)$. Set $A_{\sigma}=$ $\lim _{s} A_{\sigma, s}$. The $A_{\sigma, s}$ are columnwise recursive. Note that $\forall s \leq t\left(A_{\sigma, t}-A_{\sigma, s} \subseteq\right.$ $\left.A_{t}-A_{s}\right)$. 
Given $\sigma, s$ define the following functions:

$$
\begin{aligned}
& \text { use } \left.u(\sigma, s, x)=\min \left\{z: \Phi_{\sigma, s}\left(W_{\sigma, s}[z] ; x\right) \downarrow \text { and } \Phi_{\sigma, s}\left(V_{\sigma, s}[z] ; x\right) \downarrow\right\} \text { (and } z \leq s\right) \text {. } \\
& \text { length } l(\sigma, s)=\min \left\{x:\left(\Phi_{\sigma, s}\left(W_{\sigma, s} ; x\right) \uparrow\right) \vee\left(\Phi_{\sigma, s}\left(V_{\sigma, s} ; x\right) \uparrow\right)\right. \\
& \vee\left(\Phi_{\sigma, s}\left(W_{\sigma, s} ; x\right) \neq \Phi_{\sigma, s}\left(V_{\sigma, s} ; x\right)\right) \\
& \vee\left(\left(W_{\sigma, s}\right)^{*}[u(\sigma, s, x)] \cap\left(V_{\sigma, s}\right)^{*}[u(\sigma, s, x)] \nsubseteq\{\overrightarrow{0}\}\right) \\
& \vee\left(\left(\left(W_{\sigma, s}\right)^{*} \oplus\left(V_{\sigma, s}\right)^{*}\right)[u(\sigma, s, x)] \cap\left(R-A_{\sigma, s}\right)^{*}[u(\sigma, s, x)] \nsubseteq\{\overrightarrow{0}\}\right) \\
& \vee\left(\left(\left(W_{\sigma, s}\right)^{*} \oplus\left(V_{\sigma, s}\right)^{*}\right) \oplus\left(R-A_{\sigma, s}\right)^{*}\right)[u(\sigma, s, x)] \\
& \left.\left.\neq V_{\infty}[u(\sigma, s, x)]\right)\right\} \text {. }
\end{aligned}
$$

That is the length of agreement established at stage $s$, node $\sigma$ according to the matrix describing $N_{\sigma}$ :

maximum length. $m l(\sigma, s)=\max \{l(\sigma, t) \mid t \leq s\}$.

maximum use. $m \mu(\sigma, s)=\max \{u(\sigma, t, x) \mid t \leq s$ and $x \leq l(\sigma, t)\}$.

We are now in a position to give a decision procedure for our tree. We shall inductively define an accessible (finite) branch of length $s$, called $A C(s)$. If $\sigma \in$ $A C(s)$, we say $s$ is a $\sigma$-stage. For each $\sigma$ and $s$ we define $<(\sigma, s)$, the last $\sigma$-stage $s$ via $l s(\sigma, s)=\max \{t: t<s$ and $(t=0 \vee \sigma \in A C(t))\}$. We formally define $A C(s)$ and $r(\sigma, s)$ via

(i) for all $s, \varnothing \in A C(s)$.

(ii) For all $s$ and even nodes $\sigma, r(\sigma, s)=0$.

(iii) For all $s$ and $\sigma$ if $\operatorname{lh}(\sigma)>s$ then $r(\sigma, s)=0$ and $\sigma \notin A C(s)$. In particular $r(\sigma, 0)=0$ if $\sigma \neq \varnothing$.

(iv) For all $s$ and even nodes $\sigma$ if $\sigma \in A C(s+1)$, $\operatorname{lh}(\sigma)<s, t=l s(\sigma, s)$ and $B_{s}^{(\sigma)}-B_{t}^{(\sigma)}=\varnothing$, then $\sigma^{\wedge} 0 \in A C(s+1)$. Otherwise if $\ln (\sigma) \leq s, \sigma^{\wedge} 1 \in A C(s+1)$.

(v) For all $s$ and odd nodes $\sigma$ if $\operatorname{lh}(\sigma) \leq s$ then

(a) if $t=l s(\sigma, s)$ and $l(\sigma, s)>m l(\sigma, t)$ then $\sigma^{\wedge} 0 \in A C(s+1)$,

(b) otherwise $\sigma^{\wedge} 1 \in A C(s+1)$.

(vi) For all $s$ and odd nodes $\sigma$, if $\sigma^{\wedge} 0 \in A C(s+1)$ then set $r(\sigma, s+1)=m u(\sigma, s)$.

(vii) For all $s$ and odd nodes $\sigma$ with $\operatorname{lh}(\sigma) \leq s+1$, if $\sigma^{\wedge} 0 \notin A C(s+1)$ then $r(\sigma, s+1)=r(\sigma, s)$.

Construction.

Stage 0. $A_{0}=\varnothing$.

Stage $s+1$. Suppose $\exists \tau \in A C(s+1)$ such that $\tau$ is even, and there exists $x \in R$ such that $x \in B_{s}^{(\tau)}-A_{s}^{(\tau)}$ and $x$ is $(\tau, s+1)$-free. In this case we say $x$ requires attention via $\tau$ at stage $s+1$. Let $x_{s+1}$ be the least such $x$ (if any) and set $A_{s+1}=A_{s} \cup\left\{x_{s+1}\right\}$. If no such $x$ exists set $A_{s+1}=A_{s}$.

To complete the construction set $A=\bigcup_{s} A_{s}$.

End of construction.

DEFINITION. We define the true path $\beta$ of $T$ as follows: $\varnothing \in \beta$, and for all $\sigma$, if $\sigma \in \beta$ then $\sigma^{\wedge} 0 \in \beta$ iff $\exists^{\infty} s\left(\sigma^{\wedge} 0 \in A C(s)\right)$; otherwise $\sigma^{\wedge} 1 \in \beta$.

REMARK. (iii) The true path $\beta$ is an infinite branch of $T$ such that if $\sigma \in \beta$ then there are infinitely many stages $s$ at which $\sigma \in A C(s)$ while there are only finitely many stages $s$ at which $(\exists \tau)(\tau<\sigma$ and $\tau \nsubseteq \sigma$ and $\tau \in A C(s))$.

LEMMA 6.2. If $\sigma \in \beta$ and $\sigma$ is an even node then $\lim _{s} R(\sigma, s)=R(\sigma)$ exists and is finite. 
ProOF. Let $\sigma \in \beta$ and $t$ be such that (by Remark (iii)), $\forall s \geq t \forall \tau([\tau<\sigma$ and $\tau \nsubseteq \sigma] \rightarrow \tau \notin A C(s))$. Let $\tau \leq \sigma$. If $\tau$ is even then $r(\tau, s)=0$ for all $s$. If $\tau$ is odd then $\tau<\sigma$, as $\sigma$ is even we have

Case 1. If $\tau \nsubseteq \sigma$, then $r(\tau, s)=r(\tau, t)$ for all $s \geq t$ by (vii).

Case 2. If $\tau \subseteq \sigma$, then $\tau^{\wedge} 0<\sigma$ and $\tau^{\wedge} 0 \nsubseteq \sigma$ so $\tau^{\wedge} 0 \notin A C(s)$ for all $s \geq t$, and here by (viii) $r(\tau, s)=r(\tau, t)$ for $s \geq t$.

LEMMA 6.3. Each $x \in R$ requires attention at most finitely often.

ProOF. Let $x$ be the least element of $R$ requiring attention infinitely often. Then $x \in B-A$. Choose $t$ such that $\forall s \geq t \forall y<x$ ( $y$ does not require attention at stage $x$ ). Let $s \geq t, \tau$ be such that $x$ requires attention via $\tau$ at stage $s+1$. Then at this stage $x=x_{s+1}$.

LEMMA 6.4. $\forall e\left(B^{(e)}={ }^{*} A^{(e)}\right)$ and $A^{\prime} \equiv_{T} \varnothing^{\prime \prime}$.

Proof. Fix $e$ and let $\sigma \in \beta$ be such that $\ln (\sigma)=2 e$. Let $t$ be a stage such that $\forall s \geq t, R(\sigma, s)=R(\sigma, t)=R(\sigma)$. By construction $R(\sigma)^{*}$ is finite dimensional. For each $x \in B^{(e)}-A^{(e)}$ we may find a stage $s_{x}>t$ at which no $y<x$ requires attention. Then at each such stage $x$ must be restrained by $R(\sigma)$. However in this case, infinitely many elements of $B^{(e)}$ must occur in $(R-A)$ and so an infinite dimensional space (namely $\left\{x \in B^{(e)}-A^{(e)}\right\}$ ) is restrained by a finite dimensional one $\left.R(\sigma)^{*}\right)$. Therefore at most finitely many $x \in B^{(e)}-A^{(e)}$ are restrained from entering $A$. Finally $A^{\prime} \equiv_{T} \varnothing^{\prime \prime}$ by construction of $B$.

LEMMA 6.5. If $\sigma \in \beta$ and $\sigma$ is even, then $\sigma^{\wedge} 0 \in \beta$ iff $B^{(\sigma)}=\omega^{(\sigma)}$.

PROOF. Straightforward induction.

LEMMA 6.6. $\forall \sigma\left(\sigma \in \beta \rightarrow A_{\sigma}=A\right)$.

Proof. Consider $A^{(e)}$. Let $\tau \in \beta$ with $\operatorname{lh}(\tau)=2 e$. If $\tau^{\wedge} 0 \nsubseteq \sigma$, then $A_{\sigma}^{(\tau)}=$ $A^{(\tau)}$ by definition of $A_{\sigma}$. Suppose $\tau^{\wedge} 0 \subseteq \sigma$. By the above $\lim _{s} Q(\sigma, s)=Q(\sigma)$ exists. By definition $A_{\sigma}^{(\tau)}[Q(\tau)]=A^{(\tau)}[Q(\tau)]$. Now suppose $x \in R$ and $x \geq Q(\tau)$, then $x \in A_{\sigma}^{(\tau)}$. Since $\exists^{\infty} s\left(\tau^{\wedge} 0 \in A C(s+1)\right)$, by (iv) (of the definition of $A C$ ) $\exists^{\infty} s\left(B_{s}^{(\tau)}-B_{l s(\tau, s)}^{(\tau)} \neq \varnothing\right)$. Since $B^{(\tau)}$ is an initial segment of $R($ or $\omega), B^{(\tau)}=\omega^{(\tau)}$. By Lemma 6.5 and the fact that $x$ is $(\tau, s)$-free for infinitely many $s, x \in A^{(\tau)}$ and so $A_{\sigma}^{(\tau)}=A^{(\tau)}$.

LEMMA 6.7. Suppose $\sigma \in \beta$ is odd, $V_{\sigma} \oplus W_{\sigma} \oplus(R-A)^{*}=V_{\infty}, \Phi_{\sigma}\left(V_{\sigma}\right)=$ $\phi_{\sigma}\left(W_{\sigma}\right)=f$ and $f$ is total. Then $\sigma^{\wedge} 0 \in \beta$.

ProOF. By Lemma 6.6 $A_{\sigma}=A$, we note that $\lim _{s} l(\sigma, s)=\infty$. Thus by (iv) $\exists^{\infty} s\left(\sigma^{\wedge} 0 \in A C(s)\right)$ and so $\sigma^{\wedge} 0 \in \beta$.

LEMMA 6.8. Suppose $\sigma \in \beta$ and $\sigma$ is odd. Let $t$ be a stage such that

(i) $\forall \tau\left(\tau^{\wedge} 1 \subseteq \sigma \rightarrow A^{(\tau)}=A_{t}^{(\tau)}\right)$.

(ii) $\forall s \geq t \forall \tau([\tau<\sigma$ and $\tau \nsubseteq \sigma] \rightarrow \tau \notin A C(s))$.

Let $s, u$ be such that $t \leq s \leq u, s+1$ and $u+1$ are $\sigma^{\wedge} 0$-stages and $s+1=$ $l s\left(\sigma^{\wedge} 0, u+1\right)$. Then either

(a) $\left(V_{\sigma, s}\right)^{*}[r(\sigma, s)]=\left(V_{\sigma, u}\right)^{*}[r(\sigma, s)]$, or

(b) $\left(W_{\sigma, s}\right)^{*}[r(\sigma, s)]=\left(V_{\sigma, u}\right)^{*}[r(\sigma, s)]$. 
PROOF. Let $\sigma, t, s$ and $u$ be as above. By remark (ii) provided we were successful in $(\sigma, s+1)$ restraining between stages $s+1$ and $u+1$, the result will follow. By construction at most one number enters $A$ at any stage. Let $f$ be the least stage such that some $x<Q(\sigma, s+1)$ in $R$ receives attention (with $\sigma \leq u$ ) through $\tau$ say. Since $t \leq s<f$, no node $\rho<\sigma$ is in $A C(f)$ so $\tau \not \leq \sigma$. Therefore as, by definition of $s+1$ and $u+1, \sigma^{\wedge} 0 \notin A C(f)$ so $\sigma^{\wedge} 0 \nsubseteq \tau$. Hence $\sigma<\tau$. However by construction $r(\sigma, s+1) \leq r(\sigma, f) \leq R(\tau, f)$ contradicting the fact that $x$ is not $\sigma$-restrained (rather $\sigma$-free).

LEMMA 6.9. Suppose $V_{\sigma} \oplus W_{\sigma} \oplus(R-A)^{*}=V_{\infty}$ and $\Phi_{\sigma}\left(V_{\sigma}\right)=\Phi_{\sigma}\left(W_{\sigma}\right)=f$ and $f$ is total. Then $f$ is recursive.

Proof. By Lemma 6.6, $A_{\sigma}=A$, and by Lemma $6.7, \sigma^{\wedge} 0 \in \beta$. For each $\tau$ if $\tau^{\wedge} 1 \subseteq \sigma$ then $B^{(\tau)}$ is finite and so $A^{(\tau)}$ is finite. Let $t$ be a stage so large that

(i) $\forall \tau\left(\tau^{\wedge} 1 \subseteq \sigma \rightarrow A_{t}^{(\tau)}=A^{(\tau)}\right)$.

(ii) $\forall s \geq t \forall \tau([\tau<\sigma \wedge \tau \nsubseteq \sigma] \rightarrow \tau \notin A C(s))$.

Now by hypothesis $\lim _{s} l(\sigma, s)=\infty$. We show how to compute $\Phi_{\sigma}\left(V_{\sigma}\right)=f$ recursively.

Let $z \in V_{\infty}$. Find a stage $s \geq t$ such that $\sigma^{\wedge} 0 \in A C(s+1)$ and $z<l(\sigma, s)$. Let $y=u(\sigma, s, z)$. Then $\Phi_{\sigma, s}\left(V_{\sigma, s}[y] ; z\right)=\Phi_{\sigma, s}\left(W_{\sigma, s}[y] ; z\right)$ and $y \leq r(\sigma, s+1)$. Let $u$ be a $\sigma^{\wedge} 0$ stage with $s+1=l s(\sigma, u+1)$. By Lemma 6.8 one of $\left(V_{\sigma, s}\right)^{*}[r(\sigma, s)]$ or $\left(W_{\sigma, s}\right)^{*}[r(\sigma, s)]$ equals $\left(V_{\sigma, u}\right)^{*}[r(\sigma, u)]$ or $\left(W_{\sigma, u}\right)^{*}[r(\sigma, s)]$ respectively. E.g. $\left(V_{\sigma, s}\right)^{*}[y]$ $=\left(V_{\sigma, u}\right)^{*}[y]$. Thus

$$
\Phi_{\sigma, s}\left(V_{\sigma, s} ; z\right)=\Phi_{\sigma, s}\left(V_{\sigma, s}[y] ; z\right)=\Phi_{\sigma, u}\left(V_{\sigma, u}[y] ; z\right)=\Phi_{\sigma, s}\left(W_{\sigma, s} ; z\right)=\Phi_{\sigma, u}\left(W_{\sigma, u} ; z\right)
$$

By induction, if $s^{\prime}$ is the least stage $s^{\prime} \geq t$ with $\sigma^{\wedge} 0 \in A C(s+1)$ and $x<l(\sigma, s)$ and if $u>s^{\prime}$ is any $\sigma^{\wedge} 0$ stage, the $\Phi_{\sigma, u}\left(V_{\sigma, u} ; z\right)=\Phi_{\sigma}\left(V_{\sigma} ; z\right)$ and so $f$ is recursive, concluding the proof of Theorem 6.1.

Using a similar (but easier) construction we may also prove

THEOREM 6.10. There exists an immune co-r.e. subset $R$ of a recursive basis $B$ such that every r.e. complement of $(R)^{*}$ is strongly atomic.

Theorems (6.3) and (6.10) combine to give us a wealth of existence results as follows.

THEOREM 6.11. There exist strongly atomic $V \in L\left(V_{\infty}\right)$ of the following types:

(i) $V$ is high,

(ii) $V$ is low,

(iii) $V$ is supermaximal,

(iv) $V$ is $k$-thin for any $k \in \omega$,

(v) $V$ is nowhere simple and nonrecursive,

(vi) $V$ is super-r-maximal, but not maximal (and may be constructed to be contained in no maximal, or contained in a maximal r.e. subspace).

ProOF. (i) is immediate by (6.3). To get (ii) use Theorem 6.3 where we consider $A$ (of 6.3) as nonsimple. Then we can Sacks' split $A$ as $A_{1} \sqcup A_{2}$ with $A_{i}$ low and nonrecursive. By [DR1] there exists $V \in L\left(V_{\infty}\right)$ with $V \equiv_{T} A_{1}$ and $V \oplus(B-A)^{*}=V_{\infty}$. Then $V$ has the desired properties. (iii)-(vi) all follow by 
[Do2], who showed that if $R$ is immune and fully co-r.e., then $(R)^{*}$ automatically has r.e. complements of the desired types.

We remark that $[\mathbf{D W}]$ used strongly atomic r.e. sets to give examples of r.e. sets with very strong antisplitting properties. We do not know if the analogous properties hold in $L\left(V_{\infty}\right)$. The key to the results of the next section, though, is the use of these spaces since if $Q \oplus W=V$, then $Q \not_{T} \varnothing, W \equiv_{T} \varnothing$ implies $\left.Q\right|_{T} W$.

7. Anticupping. In this section using a fairly complicated construction via the results of $\S 6$, we shall show that any reasonable analogue of (6.2) fails. Our result is

THEOREM 7.1. There exists $V \in L\left(V_{\infty}\right)$ and $W, Q \in L\left(V_{\infty}\right)$ such that $Q \oplus W=$ $V$, and for all $Q^{\prime}, F \in L\left(V_{\infty}\right)$ if $Q^{\prime} \oplus F=V$ and if $Q^{\prime} \equiv_{T} Q$, then $d(Q) \vee d(F) \not$ $d(V)$.

We shall establish (7.1) by the next result.

THEOREM 7.2. There exists an r.e. nonrecursive basis $B$ of $V \in L\left(V_{\infty}\right)$ such that

(i) $B$ is a $W$-anticupping witness for $V$,

(ii) $d(V)$ is contiguous,

(iii) $V$ is strongly atomic.

LEMMA 7.3. $(7.2) \rightarrow(7.1)$.

ProOF. By $\S 4$, there exists $Q \in L\left(V_{\infty}\right)$ with $Q \oplus W=V$ for some $W \in L\left(V_{\infty}\right)$, and $Q \equiv_{T} D(Q) \equiv_{T} B$. Now suppose for some $Q^{\prime}, F \in L\left(V_{\infty}\right), Q^{\prime} \equiv_{T} Q$ and $Q^{\prime} \oplus F=V$ and $d\left(Q^{\prime}\right) \vee d(F)=d(V)$. Let $A(Q)=\{2 x \mid x \in Q\}$ and $A(F)=$ $\{2 x+1 \mid x \in F\}$. Then $A(Q) \sqcup A(F) \equiv_{T} V$. By contiguity $A(Q) \sqcup A(F) \equiv_{W} V$. Now, as $Q^{\prime} \oplus F=V$, by strong atomicity, as $\varnothing<_{T} Q^{\prime}<_{T} V$ (by contiguity), it follows that $F<_{T} V$. Hence $A(F) W$-cups $A(Q)$ to $V$, contradicting the fact that $B$ is $W$-anticupping witness for $V$. Hence (7.1) holds for $V$.

The remainder of this section is devoted to a proof of (7.2). Interestingly, we shall construct $V$ with a fully extendible basis. Thus, let $R=\left\{a_{0}<a_{1}<\cdots\right\}$ be an r.e. basis of $V_{\infty}$. We shall construct $A=\bigcup_{s} A_{s} \subset R$ and $B=\bigcup_{s} B_{s}$ such that $(B)^{*}=(A)^{*}$ and satisfy

$$
\begin{aligned}
& P_{2 e+1}: \bar{A} \neq R_{e}\left(\text { where } R_{e} \text { is the } e \text { th r.e. subset of } R\right) . \\
& P_{2 e}: \bar{B} \neq \omega_{e} .
\end{aligned}
$$

Strong atomicity

$$
\begin{aligned}
N_{2 e}: & \text { If } W_{e} \oplus V_{e}=(A)^{*} \text { and } \Psi_{e}\left(W_{e}\right)=\Psi_{e}\left(V_{e}\right)=f \\
& \text { and } f \text { is total, then } f \text { is recursive. }
\end{aligned}
$$

Contiguity

$$
N_{2 e+1}: \text { If } \Phi_{e}\left(M_{e}\right)=A \text { and } \Omega_{e}(A)=M_{e} \text { then } M_{e} \equiv_{W} A .
$$

Anticupping

$$
D_{e}: \text { If } \hat{\Gamma}_{e}\left(B \# Q_{e}\right)=A \text { then } A \leq_{W} Q_{e} .
$$

Here $A \# B$ denotes $\{2 x: x \in A\} \cup\{2 x+1: x \in B\}$, and $\left\langle\Psi_{e}, W_{e}, V_{e}\right\rangle$ denotes a standard enumeration of triples of $T$-reduction $\left(\Psi_{e}\right)$ and a pair of independent r.e. 
subspaces $\left(W_{e}, V_{e}\right),\left\langle\Phi_{e}, \Omega_{e}, M_{e}\right\rangle$ a standard enumeration of triples of pairs of $T$ reductions $\left(\Phi_{e}, \Omega_{e}\right)$ and r.e. sets $\left.M_{e}\right)$, and $\left\langle\hat{\Gamma}_{e}, N_{e}\right\rangle$ a standard enumeration of pairs consisting of a $W$-reduction $\left(\hat{\Gamma}_{e}\right)$ with use $\left(\gamma_{e}\right)$ and an r.e. set $Q_{e}$. The ranking is

$$
D_{0}, N_{0}, N_{1}, P_{0}, D_{1}, N_{2}, N_{3}, P_{1}, \ldots
$$

For the $N_{j}$ requirements we shall build a "confirmation tree" similar to the one used by Stob $[\mathbf{S t}]$. Let

$$
\begin{array}{r}
l(2 e+1, s)=\max \left\{x: \forall y<x\left(\Phi_{e, s}\left(M_{e, s} ; y\right)=A_{s}(y) \& \Omega_{e, s}\left(A_{s} ; y\right)=M_{e, s}(y)\right)\right\} \\
l(2 e, s)=\max \left\{x: \forall y<x\left(\Psi_{e, s}\left(V_{e, s} ; y\right)=\Psi_{e, s}\left(W_{e, s} ; y\right) \&\right.\right. \\
\left.\left.\left(W_{e, s} \oplus V_{e, s}\right)^{*}[y]=\left(A_{s}\right)^{*}[y]\right)\right\}
\end{array}
$$

Associated with the $l(2 e, s)$ will be a restraint $r(2 e, s)$, generated by the maximum element used in $l(2 e, s)$ denoted by $m u(2 e, s)$, and, as in $\S 6$, we define $y$ to be $(2 e, s)$ free if its addition to $A_{s+1}-A_{s}$ would not injure the computations involved. As this is so similar to $\S 6$, we do not mention this further, save to say similar modifications apply for the $\sigma$-strategy, and thus this is replaced by $(\sigma, s)$-free with $\ln (\sigma)=2 e$.

For the $N_{2 e+1}$ we use the confirmation notion of Stob [St]. Without the "guessing" for an element $x$ targeted for $A$, associated with some $R_{j}$ for $j>2 e+1$, if a stage $s$ occurs with

$$
\begin{cases}\text { (i) } & l(2 e+1, s)>x, \\ \text { (ii) } \quad \forall y \leq x(l(2 e+1, s))>u\left(\Phi_{e, s}\left(M_{e, s} ; y\right)\right),\end{cases}
$$

then $x$ becomes confirmed at this stage, and we cancel all lower priority followers (including, if necessary, followers of the same requirement which are smaller). Using the derived $\sigma$-strategy, this will suffice to show contiguity. (Cf. [St, AS1, DW].)

Finally, the $D_{e}$ will not be guessed, but will act like a Sacks restraint. It will be along the lines of [LS, Theorem 4.1] and will impose only $B$-restraint (not $A$-restraint). We define a restraint $\rho(e, s)$ via a marking function $\alpha(e, s)$ defined inductively as follows: $\alpha(e, 0)=0 . \alpha(e, s+1)$ is the least $n$ such that

(i) $n \leq \alpha(e, s)$ and $n \in A_{s+1}-A_{s}$,

(ii) $n=\alpha(e, s)$ and $\hat{\Gamma}_{e, s}\left(B_{s} \# Q_{e, s} ; y\right) \uparrow$ for some $y \leq n$,

(iii) $n \geq \alpha(e, s)$ and $\hat{\Gamma}_{e, s}\left(B_{s} \# Q_{e, s} ; y\right) \downarrow$ for all $y<n$; for all $y<n$,

$$
\hat{\Gamma}_{e, s}\left(B_{s} \# Q_{e, s} ; y\right)=A_{s+1}(y) \& \hat{\Gamma}_{e, s}\left(B_{s} \# Q_{e, s} ; n\right) \neq A_{s+1}(n) .
$$

Then

$$
\begin{gathered}
\rho^{\prime}(e, s+1)=1+\sum_{m \leq \alpha(e, s+1)}\left(1+\gamma_{e, s+1}(m)\right), \text { and } \\
\rho(e, s+1)=\max \left\{\rho^{\prime}(j, s+1): j \leq e\right\} .
\end{gathered}
$$

Now, the $D_{e}$ will not interact with the $P_{2 e+1}$ since it imposes no $A$-restraint. However, it will interact with the $B$-restraint, and so with the $P_{2 e}$. This will be reflected in the changing " $B$-follower associated with the $A$-follower $x$ at stage $s$," but we shall ensure that this will be finite injury for any choice of $x$.

Briefly, we initially appoint a follower $x \operatorname{targeted~for~both~} B$ and $A$. Now we do not know whether or not $\alpha(j, s) \rightarrow \infty$ for each $j \leq e$. We wait till $\alpha(j, s)>x$ and then we reset the follower $y=x$ targeted for $B$ to be $y=x+g$ where $g \in B_{s+1}-B_{s}$ 
and $g \in A_{s+1}-A_{s}$, and $x+g$ is very large. We do this for each $j \leq e$ the first time $\alpha(j, s)>x$ (that is, finitely often). This idea ensures that when $y$ is reset for the last time, once $y \in W_{e, s}$ we can set $A_{s+1}=A_{s} \cup\{x\}$ which forces $\rho(e, s+1)<y$ and thus allows us to add $y$ to $B_{s+1}-B_{s}$ to satisfy $P_{2 e+1}$.

At stage $s+1$ with $s$ a $\sigma$-stage, we shall say that $P_{2 e}$ requires attention if $B_{s} \cap W_{e, s}=\varnothing$ and

$$
P_{2 e} \text { has no } A \text {-follower with guess } \sigma \text {, or }
$$

$P_{2 e}$ has an $A$-follower $x$ with guess $\sigma$ such that the associated $B$ follower $y$ is bogus, meaning that condition (7.7) is unsatisfied:

"If we set $A_{s+1}=A_{s} \cup\{x\}$ then $y<\rho(e, s+1)$ ", or

$P_{2 e}$ has an $A$-follower $x$ with guess $\sigma$ such that the associated $B$ follower $y \in \omega_{e, s}$ and $y$ is not bogus.

We shall call (7.7) the bogus condition.

(Briefly, the idea here, is that we shall use $x$ to force the $\rho(e, s)$ to drop sufficiently to allow us to add $y$ to $B$. For a given $x$ with guess along the true path, we need only reset $y$ because of the bogus condition, finitely often.)

The $P_{2 e+1}$ will only be subject to tree conditions. We construct the priority tree as follows (following [St]). A stage $s$ is a $\sigma$-stage defined by induction on $\ln (\sigma)$ via

(i) every stage is a $\varnothing$-stage.

(ii) If $s$ is a $\sigma$-stage and $\mathrm{lh}(\sigma)=2 e$, then if $\forall t<s(t$ is a $\sigma$-stage $\rightarrow l(2 e, s)>$ $l(2 e, t)), s$ is a $\sigma^{\wedge} 0$-stage. Otherwise $s$ is a $\sigma^{\wedge} 1$-stage.

(iii) If $s$ is a $\sigma$-stage and $\operatorname{lh}(\sigma)=2 e+1$, then if $\forall t<s(t$ is a $\sigma$-stage $\rightarrow$ $l(2 e+1, s)>l(2 e+1, t))$ then $s$ is a $\sigma^{\wedge} 0$-stage. Otherwise $s$ is a $\sigma^{\wedge} 1$-stage.

Similarly, the $\sigma$-restraint $r(\sigma, s)$ for $\operatorname{lh}(\sigma)$ odd is defined by induction to drop to 0 at $\beta^{\wedge} 0=\sigma$ stages, and to be maintained at $\beta^{\wedge} 1=\sigma$ stages as in the construction of $\S 6$. The contiguity nodes are only cancellation ones, and impose no restraint.

A requirement $P_{2 e+1}$ requires attention

if it has no follower with guess $\subseteq \sigma_{s}$,

$$
\text { for some follower } x \text { with guess } \subset \sigma_{s}, \quad x \in R_{e, s} .
$$

Construction. Stage $s+1$.

Step 1 (Cancellation). Find the unique $\sigma_{s}$ of length $s$ such that $s$ is a $\sigma_{s}$-stage. Cancel all followers, etc. with guesses weaker than $\sigma_{s}$ (that is guesses $\tau \npreceq \sigma_{s}$ ).

Step 2 (Confirmation). Now for any number $x$ targeted for $A$ with guess $\sigma \leq \sigma_{s}$ and for any $e$ with $x$ not already $2 e$-confirmed, if $l h(\sigma) \geq 2 e$ and (7.4) holds for $x$, declare $x$ as $2 e$-confirmed and cancel all lower priority followers, etc. (Actually, only one follower gets confirmed by this process, all the others that might be confirmed will get cancelled.)

Step 3. Cancel any followers that have guesses weaker than $\sigma$ (for $\sigma=\sigma_{s}$ ) and are not $(\sigma, s)$-free.

Step 4. Find $f$ such that $P_{f}$ requires attention ( $f$ least). Cancel all followers etc. of $P_{j}$ for $j>f$ with guesses $\supseteq \sigma\left(\sigma \subseteq \sigma_{s}, \operatorname{lh}(\sigma)=e+1\right)$.

Case 1. $f=2 e+1$. 
Subcase (i). (7.9) holds. Find the unique $\sigma \subseteq \sigma_{s}$ with $\operatorname{lh}(\sigma)=2 e+2$. Find a fresh number $x \in R$ exceeding all computations etc., which is not $(\sigma, s)$-restrained, and appoint $x$ as a follower of $P_{2 e+1}$ with guess $\sigma$.

Subcase (ii). (7.10) holds. Now let $g$ be a fresh number in $R$ such that $g, x+g$ exceed all computations, are $(\sigma, s)$-free etc. (as in $\S 6$ ) and set

$$
A_{s+1}=A_{s} \cup\{x, g\} \quad \text { and } \quad B_{s+1}=B_{s} \cup\{x+g, g\} .
$$

$P_{2 e+1}$ is now met (forever). Cancel all followers of $P_{2 e+1}$.

Case 2. $f=2 e$.

Subcase (i). (7.5) holds. Find as above a fresh free number $x$ and appoint $x$ as an $A$-follower with associated $B$-follower $y=x$.

Subcase (ii). (7.6) holds. Find a large fresh free number $g$ (so that both $g, x+g$ are fresh and free) and reassign the $B$-follower by making $y=x+g$ the $B$-follower associated with $x$. (The previous one is no longer associated with $x$.) Set $A_{s+1}=$ $A_{s} \cup\{g\}$ and $B_{s+1}=B_{1} \cup\{g\}$.

Subcase (iii). (7.7) holds. Set $A_{s+1}=A_{s} \cup\{x\}$ and $B_{s+1}=B_{s} \cup\{y\} . P_{2 e}$ is now met. Cancel all followers of $P_{2 e}$.

End of construction.

We shall now give the verification, sketching only, because of its similarity with the literature and $\S 6$. Evidently, all the $N_{2 e}$ are met for the same reason as they were in $\S 6$. Also $(B)^{*}=(A)^{*}$ is easy to see (by induction). Because we have transported the contiguity machinery of Ambos-Spies $[\mathbf{A S 1}]$ or Stob $[\mathbf{S t}], A$ will be contiguous (see also [LS, DW]) (or perhaps recursive).

Briefly, assuming all the other requirements are met, let $\sigma$ be the guess on the true path corresponding to an $N_{2 e+1}$ requirement, and suppose $\Phi_{e}\left(M_{e}\right)=A$ and $\Omega_{e}(A)=M_{e}$ so that $\sigma=\beta^{\wedge} 0$. Let $t$ be a stage by which higher priority activity has ceased. Then any follower $x$ targeted for $A$ after stage $t$ is either cancelled, $\sigma$ confirmed or enumerated into $A$, and furthermore, $x$ must have guess $\tau$ no stronger than $\sigma$. Let $z$ be given. Let $t_{1}$ be the least $\sigma$-stage exceeding $t$ with $l(\sigma, t)>z$. Then for some $q<t_{1}, \Omega_{e, t_{1}}(A[q] ; z)=M_{e}(z)$. Let $\Delta(z)=t_{1}$. Find the least $\sigma$ stage $t_{2} \geq t_{1}$ with $A_{t_{2}}[\Delta(z)]=A[\Delta(z)]$. It will follow that $M_{e}(z)=M_{e, t_{2}}(z)$. For suppose not. This means that there are currently followers $y$ targeted for $A$ with $\Delta(z) \leq y \leq u=u\left(\Omega_{e, t_{2}}\left(A_{t_{2}} ; z\right)\right)<t_{2}$ (for otherwise $A_{t_{2}}[u]=A[u]$, new followers are large). By cancellation at $\sigma$-stages, as $y$ is not cancelled at stage $t_{2}$, it must have been appointed at $\sigma$-stage $t_{3}$ with $t_{1} \leq t_{3}<t_{2}$. As $y$ is still a follower at stage $t_{2}$ no follower $k<y$ can enter $A$ after stage $t_{3}$ before stage $t_{2}$, as any such $k$ must have higher priority. Thus $A_{t_{3}}[y]=A_{t_{2}}[y]$ and $y \leq \Delta(z)$. This contradicts the choice of $t_{2}$ as the least $\sigma$-stage with $A_{t_{2}}[\Delta(z)]=A[\Delta(z)]$. Hence $M_{e} \leq_{W} A$.

Finally $A \leq_{W} M_{e}$ as follows: go to stage $x$. If $x$ is not a follower targeted for $A$ at stage $x$ then $x \notin A$. Again we suppose $x$ is a stage where guesses stronger than $\sigma$ cease to act. Go to the least stage $t>x$ by which some follower $\geq x$ is $\sigma$-confirmed. Now if $x \notin A_{t}$ and $x$ is not cancelled at stage $t$ then $x$ is $\sigma$-confirmed at $t$. Set $u(x)=u\left(\Phi_{e, t}\left(M_{e, t} ; x\right)\right)$. It is not too difficult to see that if $t_{1}$ is the least $\sigma$-stage with $M_{e, t_{1}}[u(x)]=M_{e}[u(x)]$ then $x \in A$ iff $x \in A_{t_{1}}$.

To see that all the $P_{2 e}$ and $P_{2 e+1}$ are met, it will suffice to show that by way of induction, a follower $x$ of $P_{2 e+1}$ with guess on the true path can require attention (because of a bogus associated $B$-follower) finitely often. 
Let

$$
m(s+1)=1+\sum_{\substack{m \leq \alpha(j), j \leq e \\ \alpha(j)=\lim _{s} \alpha(j, s) \text { existing }}}\left(1+\gamma_{j, s+1}(m)\right)+1+\sum_{\substack{p \leq x, j \leq e \\ \alpha(j, s) \rightarrow \infty}}\left(1+\gamma_{j, s+1}(p)\right) .
$$

Then $\lim _{s} m(s)=m$ exists. Let $s$ be the least stage where $m(s)=m$. Now at some least $\sigma$-stage $>s$, the $B$-follower $y$ associated with $x$ will be reset to exceed $m$. Inspection of $m(s+1)$ reveals that this is the last time $y$ becomes reset. Now assuming $\bar{B}=\omega_{e}$, at some $\sigma$-stage $t>s, y$ occurs in $\omega_{e, t}$ and then will get enumerated into $B_{t+1}-B_{t}$. Hence the $P_{2 e+1}$ and the $P_{2 e}$ get met by an induction argument along the true path.

Finally all the $D_{e}$ are met. Following [LS], suppose $\hat{\Gamma}_{e}\left(B \# Q_{e}\right)=A$, and all higher priority requirements have received attention by stage $s_{0}$. To decide if $n$ is in $B$ find a stage $t>s_{0}$ such that

$$
n<\alpha(e, t), \quad Q_{e}\left[\max _{m \leq n} \gamma_{e}(m)\right]=Q_{e, t}\left[\max _{m \leq n} \gamma_{e}(m)\right] .
$$

Then $n \in A$ iff $n \in A_{t}$, else the least $m \leq n$ to enter $A-A_{t}$ will give us a disagreement to preserve forever by the way we have $\rho$-restrained $B$ (at each stage $s, z \in B_{s}-B_{s-1}$ iff $z>\rho(e, s)$ after stage $\left.s_{0}\right)$. This would contradict the fact that $\alpha(e, s) \rightarrow \infty$ and thus $\hat{\Gamma}_{e}\left(B \# Q_{e}\right)=A \rightarrow A \leq_{W} Q_{e}$. This concludes our proof of the result.

8. $m$-degrees of bases. Various splitting results for r.e. sets can be obtained by using the $m$-degrees of splittings. For example, in [DW and AS2], the authors used strongly atomic sets to get various splitting/embedding results. One of the primary reasons these results held, was that if $A_{1} \sqcup A_{2}=B_{1} \sqcup B_{2}=A$ for strongly atomic $A$, with the $A_{i}, B_{i}$ infinite and $A_{1} \equiv_{T} B_{1}$, then $A_{i} \equiv_{m} B_{i}$ and $A_{2} \equiv_{m} B_{2}$. We shall show that for no r.e. subspace is this situation possible.

Although we shall not pursue this aspect of $m$-degrees of bases, we remark that the $m$ - and 1-degrees of bases may be very useful in studying orbits of $L\left(V_{\infty}\right)$. We feel that this is so because of Guichard's classification [Gu1] of the automorphisms of $L\left(V_{\infty}\right)$, as those induced by recursive invertible semilinear transformations of $V_{\infty}$ (Such transformations must, of course, be 1-degree preserving, as we mentioned in $\S 1$.

THEOREM 8.1. Suppose $V \in L\left(V_{\infty}\right)$ and $B$ is an r.e. nonrecursive basis of $V$. Then there exists an infinite collection $\left\{C_{i} \mid i \in \omega\right\}$ of r.e. bases of $V$ such that

(i) for all $i, C_{i} \equiv_{T} B$, and,

(ii) for all $i \neq j,\left.C_{i}\right|_{m} C_{j}$.

PROOF. To simplify notation, we prove a weaker statement and indicate how the obvious modification could be supplied. We shall prove that there exist $C_{1}, C_{2}$ r.e. bases of $V$ with $C_{1} \equiv_{T} C_{2} \equiv_{T} B$ and $C_{1} \$_{m} C_{2}$. We build $C_{i}=\bigcup_{s} C_{i, s}$ in stages. Let $\left\{\phi_{i}: i \in \omega\right\}$ be a list of all partial recursive functions, and $B=\left\{b_{0}, b_{1}, \ldots\right\}$ an effective listing of $B$. We satisfy

$R_{e}$ : If $\phi_{e}$ total, there exists $x_{e}$ such that either (i) $x_{e} \in C_{1}$ and $\phi_{e}\left(x_{e}\right) \notin C_{2}$ or (ii) $x_{e} \notin C_{1}$ and $\phi_{e}\left(x_{e}\right) \in C_{2}$. 
In some ways, the construction is similar to that of Theorem 4.7 (i), in the sense that at each stage we put either $b_{0}+b_{s+1}$ or $b_{s+1}$ into $C_{i, s}$ ensuring $C_{i} \equiv_{T} B$. The selection of which alternative to choose depends on the satisfaction of the $R_{e}$. For example, if $\phi_{e, s} \downarrow$ and tells us to put $b_{s+1}$ into $C_{2}$ we put $b_{0}+b_{s+1}$ in instead. In the case where $\phi_{e}$ is total the nonrecursiveness of $B$ ensures that $R_{e}$ will be met.

Specifically, we employ a set of movable markers, $m(e)$ for $e=0,1, \ldots$ We let $m(e, s)$ denote the position of $m(e)$ at stage $s$. If $m(e, s)$ is defined then we ensure that $\phi_{e, s}(m(e, s)) \downarrow, m(e, s) \in C_{1, s}$ and $\phi_{e, s}(m(e, s)) \notin C_{2}$. At stage $s+1$, we wish to put either $b_{0}+b_{s+1}$ or $b_{s+1}$ into $C_{i, s+1}$. In particular, for all those $y=\phi_{e, s}(m(e, s))$ where $m(e, s)$ is defined we would like to keep $y$ out of $C_{2, s+1}$. This may be impossible since we must wait till $\phi_{e, s}(m(e, s)) \downarrow$ and put one of $b_{0}+b_{s+1}$ or $b_{s+1}$ into $C_{2, s+1}$ so that we injure certain requirements. We select the appropriate member of $b_{0}+b_{s+1}, b_{s+1}$ to injure the requirement of lowest overall priority where $R_{e}$ becomes injured at stage $s+1$ if $m(e, s)$ is defined and $\phi_{e, s}(m(e, s)) \in C_{2, s+1}-$ $C_{2, s}$. The key point is that if $R_{e}$ is injured at stage $s+1$, there must be some $i<e$ such that $m(i, s)$ is defined and $\phi_{i, s}(m(i, s)) \in\left\{b_{s+1}, b_{0}+b_{s+1}\right\}-\left\{\phi_{e, s}(m(e, s))\right\}$ so that $R_{i}$ forces $R_{e}$ 's injury. But then $\phi_{i, s}(m(i, s)) \in\left(C_{2, s}\right)^{*}-C_{2, s}$ so that $\phi_{i, s}(m(i, s))$ can never enter $C_{2}$ and so $R_{i}$ will be met, and never injure another requirement. Thus the number of injuries to $R_{e}$ is at most $e$ and this allows, as usual, $R_{e}$ to be met.

Construction.

Stage 0. Set $C_{1,0}=C_{2,0}=\left\{b_{0}\right\}$.

Stage $s+1$. Case 1. If there exists $j \leq s$ such that $m(j, s)$ is defined and $\phi_{j, s}(m(j, s)) \in\left\{b_{0}+b_{s+1} ; b_{s+1}\right\}$, then put one of $b_{0}+b_{s+1}, b_{s+1}$ into $C_{2, s+1}$ so as to injure the requirement of lowest priority being threatened. Search for the least $e \leq s+1$ such that

(i) $m(e, s)$ is undefined,

(ii) $\exists z\left[z \leq s+1\right.$ and $\phi_{e, s}(z) \downarrow$ and $z \in\left(C_{1, s}\right)^{*}-C_{1, s}$ and $\left.\phi_{e, s}(z) \in C_{2, s+1}\right]$,

(iii) at least one of $\phi_{e, s+1}\left(b_{s+1}\right) \downarrow$ or $\phi_{e, s+1}\left(b_{0}+b_{s+1}\right) \downarrow$. If there is no such $e$, set $C_{1, s+1}-c_{1, s} \cup\left\{b_{s+1}\right\}$. If there is such an $e$, there are two subcases.

Subcase (a). There exists $x \in\left\{b_{0}+b_{s+1}\right\}$ with $\phi_{e, s+1}(x) \downarrow$ and $\phi_{e, s}(x) \in C_{2, s+1}$. Pick the least such $x$ and set $C_{1, s+1}=C_{1, s} \cup\left(\left\{b_{0}+b_{s+1}, b_{s+1}\right\}-\{x\}\right)$.

Subcase (b). Otherwise. Pick the least $x \in\left\{b_{0}+b_{s+1}, b_{s+1}\right\}$ with $\phi_{e, s}(x) \downarrow$, put a $m(e)$ marker on it and put $x$ into $C_{1, s+1}$.

Case 2. There is no $j \leq s$ such that $m(j, s)$ is defined and $\phi_{j, s}(m(j, s)) \in$ $\left\{b_{0}+b_{s+1}, b_{s+1}\right\}$. Now look for the least $e$ satisfying (i), (ii) and (iii) of case 1 with $C_{2, s}$ replacing $C_{2, s+1}$. If no such $e$ exists set $C_{i, s+1}=C_{i, s} \cup\left\{b_{s+1}\right\}$ for $i=1,2$. If there is such an $e$ there are three subcases.

Subcase (a). $\exists x\left(x \in\left\{b_{0}+b_{s+1}, b_{s+1}\right\}\right.$ and $\phi_{e, s+1}(x) \in C_{2, s}$ and $\left.\phi_{e, s+1}(x) \downarrow\right)$. Pick the least such $x$ and set $C_{1, s+1}=C_{1, s} \cup\left(\left\{b_{0}+b_{s+1}, b_{s+1}\right\}-\{x\}\right)$. Set $C_{2, s+1}=C_{2, s} \cup\left\{b_{s+1}\right\}$.

Subcase (b). Subcase (a) does not hold, but there exists $x \in\left\{b_{0}+b_{s+1}, b_{s+1}\right\}$ such that

(i) $\phi_{e, s+1}(x) \downarrow$, and

(ii) $\phi_{e, s+1}(x) \in\left\{b_{0}+b_{s+1}, b_{s+1}\right\}$.

Pick the least such $x$, set $C_{2, s+1}=C_{2, s} \cup\left\{\phi_{e, s+1}(x)\right\}$ and put $C_{1, s+1}=C_{1, s} \cup$ $\left(\left\{b_{0}+b_{s+1}, b_{s+1}\right\}-\{x\}\right)$. 
Subcase (c) Otherwise. Let $x$ be the least such that $x \in\left\{b_{0}+b_{s+1}, b_{s+1}\right\}$ and $\phi_{e, s+1}(x) \downarrow$. Then set $C_{1, s+1}=C_{1, s} \cup\{x\}, C_{2, s+1}=C_{2, s} \cup\left\{b_{s+1}\right\}$ and put an $m(e)$ marker on.

Finally, if in either Cases 1 or 2 , any requirement $R_{j}$ is injured at stage $s+1$, we remove the $m(j)$ marker from its current position. If $R_{j}$ is not injured and $m(j, s)$ is defined set $m(j, s+1)=m(j, s)$. Now for all $e \leq s+1$, if $m(e, s+1)$ is not as yet defined but

(i) $\exists d\left(d \in C_{1, s+1}\right.$ and $\phi_{e, s+1}(d) \downarrow$ and $\left.\phi_{e, s+1}(d) \notin C_{2, s+1}\right)$ and

(ii) $\exists z\left(z \in\left(C_{1, s+1}\right)^{*}-C_{1, s+1}\right.$ and $\phi_{e, s+1}(z) \downarrow$ and $\left.\phi_{e, s+1}(z) \in C_{2, s+1}\right)$, then pick the least such $d$ and define $m(e, s+1)=d$.

Set $C_{i}=\bigcup_{s} C_{i, s}$.

End of construction.

We must now verify that (i) $C_{i}$ is a basis for $V$, (ii) $C_{i} \equiv_{T} B$ and (iii) $C_{1} \varliminf_{m} C_{2}$ for $i=1,2$. Now (i) is clear by exchange since either $b_{s+1}$ or $b_{0}+b_{s+1}$ enters $C_{i}$ at stage $s+1$. Let $i=1$. Given an oracle for $B$ to decide if $x \in C_{1}$, first ask if either $x$ or $x-b_{0}$ is in $B$. If not $x \notin C_{1}$. If so, either $x=b_{s}$ or $x-b_{0}=b_{s}$ some $s$, and we may find this by simply enumerating $B$. Thus $C_{i} \leq_{T} B$ and similarly $B \leq_{T} C_{i}$. It remains to verify (iii). Suppose $C_{1} \leq_{M} C_{2}$. Let $e$ be least such that $R_{e}$ is not met. Then by the remark preceding the construction we may find a stage $t$ such that $R_{e}$ is not injured at, or after stage $t$. We claim that failure of $R_{e}$ to be met implies $C_{1}$ is recursive. To decide if $x \in C_{1}$, find a stage $s \geq t$ where $\phi_{e, s}(x) \downarrow$ (here we use $\phi_{e}$ total as $R_{e}$ not met). Then $x \in C_{1}$ iff $x \in C_{1, s}$ for otherwise $x \in C_{1}-C_{1, s}$ implies that we can use $x$ to meet $R_{e}$.

To get the full statement of the theorem the requirements $R_{e}$ are replaced by

$$
\begin{aligned}
R_{e}^{\prime}: & \text { If } e=\langle i, j, k\rangle \text { then } i \neq j \text { and } \phi_{k} \text { total implies there exists } x_{e} \\
& \text { such that either (i) } x_{e} \in C_{i} \text { and } \phi_{e}\left(x_{e}\right) \notin C_{j} \text {, or, (ii) } x_{e} \notin C_{i} \\
& \text { and } \phi_{k}\left(x_{e}\right) \in C_{j} .
\end{aligned}
$$

We feel it is clear that an entirely similar construction will succeed in meeting the $R_{e}^{\prime}$, and leave this to the reader.

\section{REFERENCES}

[AS1] K. Ambos-Spies, Contiguous r.e. degrees, Logic Colloquium '83 (M. Richter, ed.), Lecture Notes in Math., vol. 1104, Springer-Verlag, Berlin and New York, 1984, pp. 1-37.

[AS2] _ Antimitotic recursively enumerable sets, Z. Math. Logik Grundlag. Math. 31 (1985), 461-477.

[AF] K. Ambos-Spies and P. Fejér, Degree theoretic splitting properties of recursively enumerable sets (to appear).

[AD] C. J. Ash and R. G. Downey, Decidable subspaces and recursively enumerable subspaces, J. Symbolic Logic 49 (1984), 1137-1145.

[De] J. C. E. Dekker, Countable vector spaces with resursive operations, part I, J. Symbolic Logic 34 (1969), 363-387.

[Do1] R. G. Downey, On a question of A. Retzlaff, Z. Math. Logik Grundlag. Math. 29 (1983), 379-384.

[Do2] _ Co-immune subspaces and complementation in $V_{\infty}$, J. Symbolic Logic 49 (1984), 528538.

[Do3] _ A note on decomposition of recursively enumerable subspaces, Z. Math. Logik Grundlag. Math. 30 (1984), 295-302.

[Do4] _ The degrees of r.e. sets without the universal splitting property, Trans. Amer. Math. Soc. 291 (1985), 337-351. 
[DR1] R. G. Downey and J. B. Remmel, The universal complementation property, J. Symbolic Logic 49 (1984), 1125-1136.

[DR2] _ Effective algebras and relational systems: coding properties, in preparation.

[DR3] _ Classification of degree classes associated with r.e. subspaces, preprint, 1986.

[DS] R. G. Downey and M. Stob, Structural interactions of the recursively enumerable $T$ - and $W$ degrees, Ann. Pure Appl. Logic 31 (1986), 205-236.

[DW] R. G. Downey and L. V. Welch, Splitting properties of r.e. sets and degrees, J. Symbolic Logic 51 (1986), 88-109.

[Gu1] D. Guichard, Automorphisms of substructure lattices in effective algebra, Ann. Pure Appl. Logic 25 (1983), 47-58.

[Gu2] _ A note on $r$-maximal subspaces of $V_{\infty}$, Ann. Pure Appl. Logic 26 (1984), 1-9.

[Ha] L. Harrington, On Cooper's proof of a theorem of Yates. I and II, handwritten notes, 1976.

[KR] I. Kalantari and J. B. Remmel, Degrees of recursively enumerable topological spaces, J. Symbolic Logic 48 (1983), 610-622.

[KRt] I. Kalantari and A. Retzlaff, Maximal vector spaces under automorphisms of the lattice of recursively enumerable vector spaces, J. Symbolic Logic 42 (1977), 481-491.

[La1] A. H. Lachlan, Lower bounds for pairs of recursively enumerable degrees, Proc. London Math. Soc. 16 (1966), 537-567.

[La2] _ Decomposition of recursively enumerable degrees, Proc. Amer. Math. Soc. 79 (1980), 629-634.

[Ld] R. Ladner, A completely mitotic nonrecursive recursively enumerable degree, Trans. Amer. Math. Soc. 184 (1973), 479-507.

[Ls] R. Ladner and L. Sasso, The weak truth table degrees of recursively enumerable sets, Ann. Math. Logic 4 (1975), 429-448.

[LR1] M. Lerman and J. B. Remmel, The universal splitting property. I, Logic Colloquium ' 80 (Van Dalen, Lascar and Smiley, eds.), Studies in Logic, vol. 108, North-Holland, New York, 1982, pp. 181-209.

[LR2] _ , The universal splitting property. II, J. Symbolic Logic 49 (1984), 137-150.

[MN1] G. Metakides and A. Nerode, Recursion theory and algebra, Algebra and Logic (J. N. Crossley. ed.), Lecture Notes in Math., vol. 450, Springer-Verlag, Berlin and New York, 1975, pp. 209-219.

[MN2] _ Recursively enumerable vector spaces, Ann. Math. Logic 11 (1977), 147-171.

[MS] M. Morley and R. I. Soare, Boolean algebras, splitting properties and $\Delta_{2}^{0}$ sets, Fund. Math. 90 (1975), 45-52.

[NR1] A. Nerode and J. B. Remmel, Recursion theory on matroids, Patras Logic Sympos. 1980 (G. Metakides, ed.), North-Holland, Amsterdam, 1982, pp. 41-67.

[NR2] _ Recursion theory on matroids. II, Southeast Asian Conf. on Logic (C.T. Chong and M. J. Wicks, eds.), Studies in Logic, vol. 111, North-Holland, Amsterdam, 1983, pp. 133-184.

[NR3] - A survey of lattices of r.e. substructures, Recursion Theory (A. Nerode and R. Shore, eds.), Proc. Sympos. Pure Math., vol. 42, Amer. Math. Soc., Providence, R. I., 1985, pp. $323-375$.

[Od] P. Odifreddi, Strong reducibilities, Bull. Amer. Math. Soc. (N.S.) 4 (1981), 37-86.

[Re1] J. B. Remmel, On r.e. and co-r.e. vector spaces with nonextendible bases, J. Symbolic Logic 45 (1980), 20-34.

[Re2] _ Recursion theory on algebraic structures with an independent set, Ann. Math. Logic 18 (1980), 153-191.

[Rt] A. Retzlaff, Direct summands of r.e. vector spaces, Z. Math. Logik Grundlag. Math. 25 (1979), 363-372.

[Ro] H. J. Rogers, Theory of recursive functions and effective computability, McGraw-Hill, New York, 1967.

[Sa] G. E. Sacks, On the degrees less than $\mathbf{0}^{\prime}$, Ann. of Math. 77 (1963), 211-231.

[Sh] R. A. Shore, Controlling the dependence degree of a recursively enumerable vector space, J. Symbolic Logic 43 (1978), 13-22.

[So1] R. I. Soare, The infinite injury priority method, J. Symbolic Logic 41 (1976), 513-530.

[So2] _ Recursively enumerable sets and degrees, Springer-Verlag (to appear). 
[So3] _ Tree arguments in recursion theory and the $\mathbf{0}^{\prime \prime \prime}$-priority method, Recursion Theory (A. Nerode and Shore, eds.), Proc. Sympos. Pure Math., vol. 42, Amer. Math. Soc., Providence, R. I., 1985, pp. 53-106.

[St] M. Stob, Wtt-degrees and T-degrees of r.e. sets, J. Symbolic Logic 48 (1983), 125-134.

[Y] C. E. M. Yates, A minimal pair of recursively enumerable degrees, J. Symbolic Logic 31 (1966), 159-168.

Department of Mathematics, Victoria University of Wellington, Private BAG, WELLINGTON, NEW ZEALAND

Department of Mathematics, University of California at SAN Diego, La Jolla, CALIFORNiA 92093

Department of Mathematics, Western Illinois University, Macomb, Illinois 61455 